\title{
SEMIDISCRETIZATION AND LONG-TIME ASYMPTOTICS OF NONLINEAR DIFFUSION EQUATIONS
}

\author{
JOSÉ A. CARRILLO*, MARCO DI FRANCESCO ${ }^{\dagger}$, AND MARIA P. GUALDANI ${ }^{\ddagger}$
}

\begin{abstract}
We review several results concerning the long-time asymptotics of nonlinear diffusion models based on entropy and mass transport methods. Semidiscretization of these nonlinear diffusion models are proposed and their numerical properties analyzed. We demonstrate the long-time asymptotic results by numerical simulation and we discuss several open problems based on these numerical results. We show that for general nonlinear diffusion equations the long-time asymptotics can be characterized in terms of fixed points of certain maps which are contractions for the euclidean Wasserstein distance. In fact, we propose a new scaling for which we can prove that this family of fixed points converges to the Barenblatt solution for perturbations of homogeneous nonlinearities near zero.
\end{abstract}

Key words. Nonlinear diffusion, long-time asymptotics, mass transport methods.

AMS subject classifications. 35B40 35K55 35K65

\section{Introduction}

The fine description of long-time asymptotics for nonlinear diffusion equations has attracted the attention of many researchers in the last few years. This revival has been devoted to new ideas brought up in the subject from different communities: the entropy approach from kinetic theory having its roots in the famous $H$-theorem for the Boltzmann equation [18, 27], the optimal mass transport theory giving a geometric point of view of these equations based on suitable metrics in the space of probability measures $[29,17,1,2]$ and variational techniques related to new Gagliardo-Nirenberg inequalities [21].

This review is intended to show some recent topics of research concerning longtime asymptotics of equations of the form

$$
\frac{\partial u}{\partial t}=\operatorname{div}(u \nabla V+\nabla f(u)), \quad x \in \mathbb{R}^{N}, \quad t>0,
$$

in which $V(x) \in W_{\text {loc }}^{1,1}\left(\mathbb{R}^{N}\right)$ is positive and $f(u)$ verifies

(F1) $f$ belongs to $C([0,+\infty)) \cap C^{1}((0,+\infty))$,

(F2) $f$ strictly increasing such that $f(0)=0$ and $f^{\prime}(u)>0$ for all $u>0 . f^{-1}$ is Hölder continuous of order $\theta$ with $0<\theta<1$. Moreover the function $h(u):=$ $\int_{1}^{u} \frac{f^{\prime}(s)}{s} d s, u>0$ belongs to $L^{1}((0,+\infty))$ with the property $h(+\infty)=+\infty$.

This family of equations includes nonlinear Fokker-Planck equations where $V(x)$ is assumed to be confining (see next section for details) and general nonlinear diffusion equations where $V(x)=0$. The Cauchy problem for general nonlinear diffusion equations is well-posed by classical results $[9,7]$ and for the nonlinear Fokker-Planck equations we refer to [16] and the references therein. We remark that solutions to (1.1) stay nonnegative for nonnegative initial data and we will reduce to nonnegative solutions in the rest.

*ICREA (Institució Catalana de Recerca i Estudis Avançats) and Departament de Matemàtiques, Universitat Autònoma de Barcelona, E-08193 Bellaterra, Spain (carrillo@mat.uab.es).

${ }^{\dagger}$ Dipartimento di Matematica, Sezione di Matematica per l'Ingegneria, Università di L'Aquila, I-67100 L'Aquila, Italy (difrance@univaq.it).

¥ICES, The University of Texas at Austin, 1 University Station C0200 Austin, Texas 78712, USA (gualdani@ices.utexas.edu). 
Nonlinear diffusion equations without confinement $V(x)=0$ are expected to diffuse as $t \rightarrow \infty$, and thus, solutions should vanish as $t \rightarrow \infty$ with an expansion of their support or their tails depending whether the diffusion is slow or fast. On the contrary, nonlinear Fokker-Planck equations are expected to stabilize towards an steady state defined by setting the flux to zero in (1.1). The rigorous proof of this stabilization was done in $[29,16]$ in $L^{1}$ by using an entropy-entropy production approach. The stationary state was characterized as the unique minimizer in the space of integrable functions with given mass of a suitable functional that we call entropy. This entropy functional was then proved to be a Lyapunov functional for the equation and thus, the study of its evolution gave the desired convergence rate. We refer to $[18,29,16,27]$ for details. Moreover, generalized Log-Sobolev inequalities were obtained in [16] relating the entropy to the entropy production.

Both equations share remarkable properties with respect to Wasserstein distances for probability measures. These distances are well-known in the probability community since probability measure spaces with suitable bounded moments endowed with these distances become complete metric spaces. The remarkable property of the family of equations (1.1) is that assuming that $V(x)$ is convex, their flow map is a global contraction for the Euclidean Wasserstein distance [29, 17, 1, 2]. Moreover, in the one-dimensional case, equations (1.1) under convexity assumptions on $V(x)$, are contractions for all Wasserstein distances [19, 14]. Recently, J. L. Vázquez [37] has shown a very nice counterexample proving that this result is not true for large index in the Wasserstein distance in any other dimension. However, it is an open problem to show if it is asymptotically, for large times, true.

Nonlinear Fokker-Planck equations with confining potential $V(x)=\frac{1}{2}|x|^{2}$ and homogeneous nonlinearity $f(u)=u^{m}$ are equivalent through an explicit change of variables to the nonlinear diffusion equation with $V(x)=0$ and $f(u)=u^{m}$ and therefore, the study of their long-time behavior is equivalent too. In fact, the stabilization towards equilibrium of the nonlinear Fokker-Planck equation translates into a self-similar behavior as $t \rightarrow \infty$ for the nonlinear diffusion equation, in the sense that all solutions resemble a self-similar profile as $t \rightarrow \infty$. This self-similar profile is the well-known Barenblatt profile for homogenous nonlinear diffusions.

We will show in Section 2 that the semidiscretization of equations (1.1) based on the implicit Euler scheme formally preserves the non-increasing behavior of the entropy functional. Moreover, in Section 2.2 we will analyse how to discretize nonlinear diffusion Fokker-Planck equations in order to avoid numerical instabilities arising due to the stabilization towards equilibria with degenerate diffusions. This numerical scheme enjoying entropy decay does not preserve theoretically the contraction of Wasserstein metrics at a discrete level. Let us mention that one-dimensional schemes have recently been proposed in [22] preserving the contraction of metrics.

In Section 3, the contraction of Wasserstein distances in one space dimension for general nonlinear diffusion equations is used in the case of asymptotically homogeneous nonlinearities to obtain a bound on the expansion rate of the support of solutions. They will behave basically like the Barenblatt profile corresponding to the exponent to which the nonlinearity resembles for small values of $u$. This result is already known since the work of J. L. Vázquez [34] but here we will give a original proof related to the recent result in [14].

Although several qualitative properties of the solutions for general nonlinear diffusion equations have been obtained [25], there is no result concerning asymptotic profiles of general diffusion equations except in the case of power-like behavior for 
small values of $u$. One of the objectives of this review is to summarize the results recently obtained in [11], in which asymptotic profiles for general nonlinear diffusion equations are obtained and characterized by being fixed points of suitable contraction maps in Wasserstein distances. This result will be explained in detailed in Section 4 . Moreover, we will try to elucidate numerically the behavior of these asymptotic profiles as $t \rightarrow \infty$ using the entropy preserving scheme introduced in Section 2.

Finally, we propose in Section 5 an alternative scaling to the one introduced in [11] to characterize again asymptotic profiles by means of a sequence of fixed points whose convergence for large times can be studied at least in the case of asymptotic homogeneity. In order to do that, continuity arguments with respect to the nonlinearity developed in [7] become relevant. This results shows that the large time limit of the asymptotic profile for homogeneous nonlinearities stabilizes towards the corresponding Barenblatt profile.

\section{Semidiscretization of nonlinear diffusion equations}

We consider the general non-linear Fokker-Planck equation

$$
\begin{gathered}
\frac{\partial u}{\partial t}=\operatorname{div}(u \nabla V+\nabla f(u)), \quad x \in \mathbb{R}^{N}, \quad t>0, \\
u(x, 0)=u_{I}(x), \quad x \in \mathbb{R}^{N},
\end{gathered}
$$

where $0 \leq u_{I} \in L^{1}\left(\mathbb{R}^{N}\right) \cap L^{\infty}\left(\mathbb{R}^{N}\right)$. A potential $V \geq 0$ will be called confining if it satisfies:

(V1) $V$ a convex function and $V(x) \longrightarrow+\infty$ as $|x| \longrightarrow+\infty$.

Much is already known for the problem (2.1), (2.2) about existence and uniqueness (see $[15,16]$ and references therein). Let us denote by $\left(H^{1}\right)^{*}$ the dual space of $H^{1}$.

TheOREM 2.1. There exists $u \in L^{\infty}\left(0, T, L^{\infty}\left(\mathbb{R}^{N}\right)\right) \cap L^{\infty}\left(0, T, L^{1}\left(\mathbb{R}^{N}\right)\right)$ nonnegative with $f(u) \in L^{2}\left(0, T, H^{1}\left(\mathbb{R}^{N}\right)\right) \cap L^{\infty}\left(0, T, L^{\infty}\left(\mathbb{R}^{N}\right)\right)$ and $\frac{\partial u}{\partial t} \in L^{2}\left(0, T, H^{1^{*}}\left(\mathbb{R}^{N}\right)\right)$ fulfilling

$$
\int_{0}^{T}\left\langle\frac{\partial u}{\partial t}, \Phi\right\rangle_{H^{1 *}, H^{1}} d t=-\int_{0}^{T} \int_{\mathbb{R}^{N}} \nabla \Phi \cdot(u \nabla V+\nabla f(u)) d x d t
$$

for all compactly supported $\Phi \in C^{\infty}\left(\mathbb{R}^{N} \times[0, T]\right)$.

The proof of the above theorem is worked out in the forthcoming paper [15] in case the potential is a positive function, not necessarily convex, such that $\|\Delta V\|_{L^{\infty}\left(\mathbb{R}^{d}\right)}$ is finite. Problem (2.1), (2.2) has the mass conservation property, i.e.,

$$
\|u(\cdot, t)\|_{L^{1}\left(\mathbb{R}^{N}\right)}=\left\|u_{I}(\cdot)\right\|_{L^{1}\left(\mathbb{R}^{N}\right)},
$$

for all $t>0$ and it is also known that its solution $u$ converges exponentially fast to the stationary state $u_{\infty}$, solution of the problem

$$
u_{\infty} \nabla V+\nabla f\left(u_{\infty}\right)=0, \quad\left\|u_{\infty}(\cdot)\right\|_{L^{1}\left(\mathbb{R}^{N}\right)}=\left\|u_{I}(\cdot)\right\|_{L^{1}\left(\mathbb{R}^{N}\right)} .
$$

The proof of this result is based on the so-called entropy dissipation method, in which the convergence towards equilibrium is concluded using the time monotonicity of the physical entropy

$$
E(u(t)):=\int_{\mathbb{R}^{N}}[u(x, t) V(x)+\phi(u(x, t))] d x
$$


where $\phi$ is a strictly convex function determined by

$$
\phi^{\prime \prime}(u)=\frac{f^{\prime}(u)}{u}, \quad \phi^{\prime}(1)=0, \quad \phi(0)=0,
$$

(see $[18,29,16]$ for details).

As already mentioned in the introduction, in the special case $V(x)=\frac{1}{2}|x|^{2}$ and $f(u)=u^{m}$, if $N(m-1)+2>0$, there exists a time-depending scaling which transforms equation (2.1) into the diffusion equation

$$
\begin{aligned}
& \frac{\partial v}{\partial t}=\Delta v^{m}, \quad x \in \mathbb{R}^{N}, \quad t>0, \\
& v(x, 0)=u_{I}(x) .
\end{aligned}
$$

This result allows us to translate any result on the asymptotic behavior of equation (2.1), if $V(x)=\frac{1}{2}|x|^{2}$ and $f(u)=u^{m}$, into results of asymptotic behavior in time of equation (2.6). In fact it has been shown [18] that the similarity solution of (2.6)

$$
B(|x|, t)=t^{-\frac{N}{\lambda}}\left(C-\frac{(m-1)}{2 m \lambda}|x|^{2} t^{-\frac{2}{\lambda}}\right)_{+}^{\frac{1}{m-1}}
$$

where $\lambda:=N(m-1)+2$, evaluated at the time $t=\frac{1}{\lambda}$, coincides with the unique compactly supported equilibrium state $u_{\infty}$ of $(2.1)$.

In this work we consider a fully implicit Euler semidiscretization for equation (2.1)

$$
\frac{u_{k}(x)-u_{k-1}(x)}{\Delta t}=\operatorname{div}\left(u_{k}(x) \nabla V(x)+\nabla f\left(u_{k}(x)\right)\right),
$$

where $u_{k}(x)$ denotes the approximation $u(x, k \Delta t), k \in \mathbb{N}$. The main properties of the above semidiscretization are proved in [15] and summarized in Theorem 2.3 for which we show a formal proof. We start by stating the result of existence shown in [15] for the semidiscrete problem in case the potential is a positive function, not necessarily convex, such that $\|\Delta V\|_{L^{\infty}\left(\mathbb{R}^{d}\right)}$ is finite.

TheOREM 2.2. For each $k \in \mathbb{N}$ there exists a nonnegative weak solution $u_{k} \in L^{2}\left(\mathbb{R}^{N}\right)$ with $f\left(u_{k}\right) \in H^{1}\left(\mathbb{R}^{N}\right)$ of (2.8) fulfilling

$$
\int_{\mathbb{R}^{N}} \nabla \psi \cdot \nabla f\left(u_{k}\right) d x=-\int_{\mathbb{R}^{N}} u_{k} \nabla \psi \cdot \nabla V d x-\frac{1}{\Delta t} \int_{\mathbb{R}^{N}} \psi\left(u_{k}-u_{k-1}\right) d x
$$

for all $\psi \in H^{1}\left(\mathbb{R}^{N}\right)$ with compact support.

The basic property of the above numerical scheme is the decay of the relative entropy

$$
E\left(u \mid u_{\infty}\right):=\int_{\mathbb{R}^{N}}\left[\phi(u)-\phi\left(u_{\infty}\right)-\phi^{\prime}\left(u_{\infty}\right)\left(u-u_{\infty}\right)\right] d x .
$$

Let us also remember that for general nonlinear Fokker-Planck equations the entropy (2.4) and the relative entropy (2.10) satisfy the following inequality

$$
E(u)-E\left(u_{\infty}\right) \geq E\left(u \mid u_{\infty}\right),
$$

being equal if and only if $u_{\infty}$ is positive everywhere (see [16, Proposition 5]). Moreover the difference can be explicitly written as

$$
\left.E(u)-E\left(u_{\infty}\right)-E\left(u \mid u_{\infty}\right)=\int_{\mathbb{R}^{N}}\left[V(x)+\phi^{\prime}\left(u_{\infty}(x)\right)\right]\left(u-u_{\infty}\right)\right) d x .
$$


In the rest, we assume furthermore that $V$ is a uniformly convex function, which means

$$
\xi \operatorname{Hess}(V(x)) \xi^{T} \geq \alpha|\xi|^{2}, \quad \forall \xi, x \in \mathbb{R}^{N}, \quad \alpha>0
$$

The following results holds:

THEOREM 2.3. For $k \in \mathbb{N}$ let $u_{k}$ be a recursively defined nonnegative weak solution of (2.8) in the sense of Theorem 2.2. It holds

$$
E\left(u_{k}\right)-E\left(u_{\infty}\right) \leq\left(E\left(u_{I}\right)-E\left(u_{\infty}\right)\right)(1+2 \alpha \Delta t)^{-k}, \quad k \in \mathbb{N},
$$

where $u_{\infty}$ describes the equilibrium function, solution to (2.3).

Proof. Here we only recall the main formal ideas behind the proof given in [15]. The proof makes use of this line of arguments by a suitable and technical approximation in bounded domains and regularization of the initial data, we refer to [15] for further details. Let

$$
D\left(u_{k}\right):=\int_{\mathbb{R}^{N}} u_{k}\left|\nabla V+\nabla \phi^{\prime}\left(u_{k}\right)\right|^{2} d x,
$$

be the entropy production for the functional $E\left(u_{k} \mid u_{\infty}\right)$; the generalized Log-Sobolev inequality [16, Theorem 17] asserts that

$$
E\left(u_{k} \mid u_{\infty}\right) \leq E\left(u_{k}\right)-E\left(u_{\infty}\right) \leq \frac{1}{2 \alpha} D\left(u_{k}\right) \quad \forall k \in \mathbb{N},
$$

using the uniform convexity of the potential $V(2.12)$. From the convexity of $\phi$, it follows

$$
\begin{aligned}
E\left(u_{k} \mid u_{\infty}\right) \geq & \int_{\mathbb{R}^{N}} \phi^{\prime}\left(u_{k+1}\right)\left(u_{k}-u_{k+1}\right)+\phi\left(u_{k+1}\right)-\phi\left(u_{\infty}\right)-\phi^{\prime}\left(u_{\infty}\right)\left(u_{k}-u_{\infty}\right) d x \\
= & \int_{\mathbb{R}^{N}} \phi\left(u_{k+1}\right)-\phi\left(u_{\infty}\right)-\phi^{\prime}\left(u_{\infty}\right)\left(u_{k+1}-u_{\infty}\right) \\
& +\int_{\mathbb{R}^{N}} \phi^{\prime}\left(u_{\infty}\right)\left(u_{k+1}-u_{k}\right)+\phi^{\prime}\left(u_{k+1}\right)\left(u_{k}-u_{k+1}\right) d x \\
= & E\left(u_{k+1} \mid u_{\infty}\right)+\int_{\mathbb{R}^{N}}\left[\phi^{\prime}\left(u_{\infty}\right)-\phi^{\prime}\left(u_{k+1}\right)\right]\left(u_{k+1}-u_{k}\right) d x .
\end{aligned}
$$

Now, using (2.11) we get

$$
\begin{aligned}
E\left(u_{k}\right)-E\left(u_{\infty}\right) \geq & E\left(u_{k+1} \mid u_{\infty}\right)+\int_{\mathbb{R}^{N}}\left[\phi^{\prime}\left(u_{\infty}\right)-\phi^{\prime}\left(u_{k+1}\right)\right]\left(u_{k+1}-u_{k}\right) d x \\
& +\int_{\mathbb{R}^{N}}\left[V+\phi^{\prime}\left(u_{\infty}\right)\right]\left(u_{k}-u_{\infty}\right) d x \\
= & E\left(u_{k+1} \mid u_{\infty}\right)-\int_{\mathbb{R}^{N}}\left[V+\phi^{\prime}\left(u_{k+1}\right)\right]\left(u_{k+1}-u_{k}\right) d x \\
& +\int_{\mathbb{R}^{N}}\left[V+\phi^{\prime}\left(u_{\infty}\right)\right]\left(u_{k}-u_{\infty}\right) d x+\int_{\mathbb{R}^{N}} \phi^{\prime}\left(u_{\infty}\right)\left(u_{k+1}-u_{k}\right) d x \\
& +\int_{\mathbb{R}^{N}} V\left(u_{k+1}-u_{k}\right) d x
\end{aligned}
$$




$$
\begin{aligned}
= & E\left(u_{k+1} \mid u_{\infty}\right)+\int_{\mathbb{R}^{N}}\left[V+\phi^{\prime}\left(u_{\infty}\right)\right]\left(u_{k+1}-u_{\infty}\right) d x \\
& -\int_{\mathbb{R}^{N}}\left[V+\phi^{\prime}\left(u_{k+1}\right)\right]\left(u_{k+1}-u_{k}\right) d x \\
= & E\left(u_{k+1}\right)-E\left(u_{\infty}\right)-\int_{\mathbb{R}^{N}}\left[V+\phi^{\prime}\left(u_{k+1}\right)\right]\left(u_{k+1}-u_{k}\right) d x .
\end{aligned}
$$

Therefore, from (2.8) after integration by parts, we deduce

$$
\int_{\mathbb{R}^{N}}\left[V+\phi^{\prime}\left(u_{k+1}\right)\right]\left(u_{k+1}-u_{k}\right) d x=-\Delta t \int_{\mathbb{R}^{N}} u_{k+1}\left|\nabla V+\nabla \phi^{\prime}\left(u_{k+1}\right)\right|^{2} d x,
$$

and thus,

$$
E\left(u_{k}\right)-E\left(u_{\infty}\right) \geq E\left(u_{k+1}\right)-E\left(u_{\infty}\right)+\Delta t D\left(u_{k+1}\right) .
$$

From inequality (2.13) it holds

$$
E\left(u_{k}\right)-E\left(u_{\infty}\right) \geq(1+2 \alpha \Delta t)\left(E\left(u_{k+1}\right)-E\left(u_{\infty}\right)\right),
$$

recursively we conclude

$$
E\left(u_{k}\right)-E\left(u_{\infty}\right) \leq(1+2 \alpha \Delta t)^{-k}\left(E\left(u_{I, m}\right)-E\left(u_{\infty}\right)\right)
$$

finishing the proof.

Let us point out that previous lemma was already observed in the linear case in [3] with a proof simplified by the fact $V(x)+\phi^{\prime}\left(u_{\infty}(x)\right)=C$ for all $\in \mathbb{R}^{N}$ for linear diffusions. It is the discrete version of the exponential decay with rate $2 \alpha$ in the continuous case obtained in $[16,18,29,21]$. In the case of general nonlinear diffusion equations we have also a decay estimate for the corresponding entropy.

COROllary 2.4. Let $v_{k}$ be solution of (2.8) with $V \equiv 0$ and

$$
\mathcal{E}(k):=\int_{\mathbb{R}^{N}} \phi\left(v_{k}(x)\right) d x .
$$

For all $k \in \mathbb{N}$ it holds $\mathcal{E}(k) \geq \mathcal{E}(k+1)$.

Proof. From equations (2.8) and (2.5) we get

$$
\begin{aligned}
\mathcal{E}(k)-\mathcal{E}(k+1) & =\int_{\mathbb{R}^{N}} \phi\left(v_{k}\right)-\phi\left(v_{k+1}\right) d x=\int_{\mathbb{R}^{N}} \phi^{\prime}\left(v_{k+1}\right)\left(v_{k}-v_{k+1}\right) d x \\
& =-\Delta t \int_{\mathbb{R}^{N}} \phi^{\prime}\left(v_{k+1}\right) \operatorname{div}\left(\nabla f\left(v_{k+1}\right)\right) d x \\
& =\Delta t \int_{\mathbb{R}^{N}} \frac{f^{\prime}\left(v_{k+1}\right)^{2}}{v_{k+1}}\left|\nabla v_{k+1}\right|^{2} d x,
\end{aligned}
$$

finishing the proof.

Let us mention that semidiscretizations derived from a discrete variational scheme based on the Wasserstein distance was introduced in the linear case in [23] and generalized to the nonlinear case in $[1,2]$ and the references therein. This semidiscretization also verifies the decay of the entropy stated in Corollary 2.4. Let us show some numerical results related to problem (2.6) and (2.1) in the case $f(u):=u^{m}$ for some $m>1$. 
2.1. The porous medium equation. We introduce the fully discretization of equation (2.6) in a uniform grid using central finite differences in space to obtain:

$$
\frac{v_{k}(i)-v_{k-1}(i)}{\Delta t}=D^{+} D^{-}\left(f\left(v_{k}(i)\right)\right), \quad k \in \mathbb{N}, \quad i=1, \ldots, M,
$$

where $D^{+}$and $D^{-}$are the standard forward and backward first order finite difference operators, defined for any discretized function $(z(i))_{i=1, \ldots, M}$ as follows

$$
\begin{array}{ll}
D^{+} z(i):=\frac{z(i+1)-z(i)}{\Delta x}, & i=1, \ldots, M-1, \\
D^{-} z(i):=\frac{z(i)-z(i-1)}{\Delta x}, & i=2, \ldots, M .
\end{array}
$$

The resulting nonlinear system of equations is iteratively solved by Newton's method at each time step. Time stepping is set to constant.

(a)

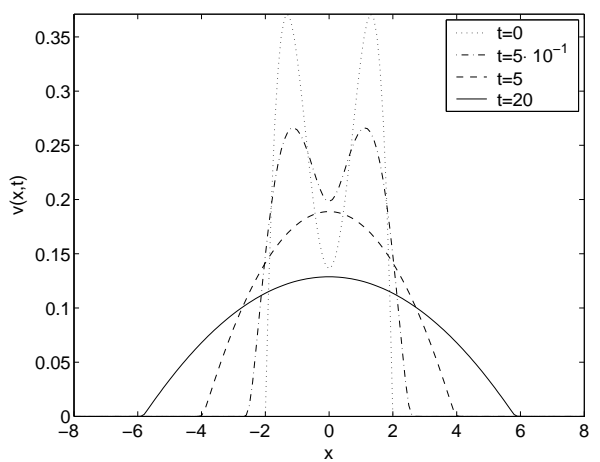

(b)

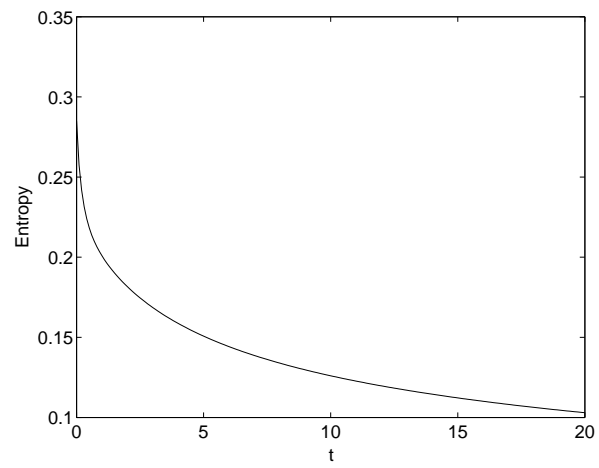

FIG. 2.1. Numerical results and entropy decay for (2.6) in case $m=2$ with initial condition (2.16): (a) time evolution of $v(x, t)$, (b) time evolution of $\mathcal{E}(t)$.

Figure 2.1 shows numerical results for $f(v):=v^{2}$ with initial data

$$
v_{I}(x)= \begin{cases}\frac{2\left[\left(4-x^{2}\right)-3.9 \cos \left(\frac{\pi}{4} x\right)\right]}{\left\|2\left[\left(4-x^{2}\right)-3.9 \cos \left(\frac{\pi}{4} x\right)\right]\right\|_{L^{1}(-2,2)}} & \text { if }-2 \leq x \leq 2, \\ 0 & \text { otherwise. }\end{cases}
$$

Let us point out that the expected Barenblatt asymptotic profile (2.7) is fixed by mass conservation

$$
\int_{\mathbb{R}} B(|x|, t) d x=\int_{\mathbb{R}} v_{I}(x) d x .
$$

The results show the convergence to the self-similar profile given by the Barenblatt profile as $t \rightarrow \infty$ and the decreasing character of the entropy. Note that in this case the decay rate of the entropy is not exponential but rather algebraic. In fact, for the porous medium equation the entropy becomes the $L^{m}$-norm of the solution that decays like $\frac{m-1}{m} \lambda$ due to the $L^{1}-L^{\infty}$ effect $[4,38]$

$$
\|v(\cdot, t)\|_{L^{\infty}\left(\mathbb{R}^{N}\right)} \leq C t^{-\frac{N}{N(m-1)+2}}\left\|v_{I}\right\|_{L^{1}\left(\mathbb{R}^{N}\right)}^{\frac{2}{N(m)+2}} .
$$


In Figure 2.2 the numerical approximations of the two dimensional porous medium equation with $f(v)=v^{3}$ together with the entropy decay are plotted.
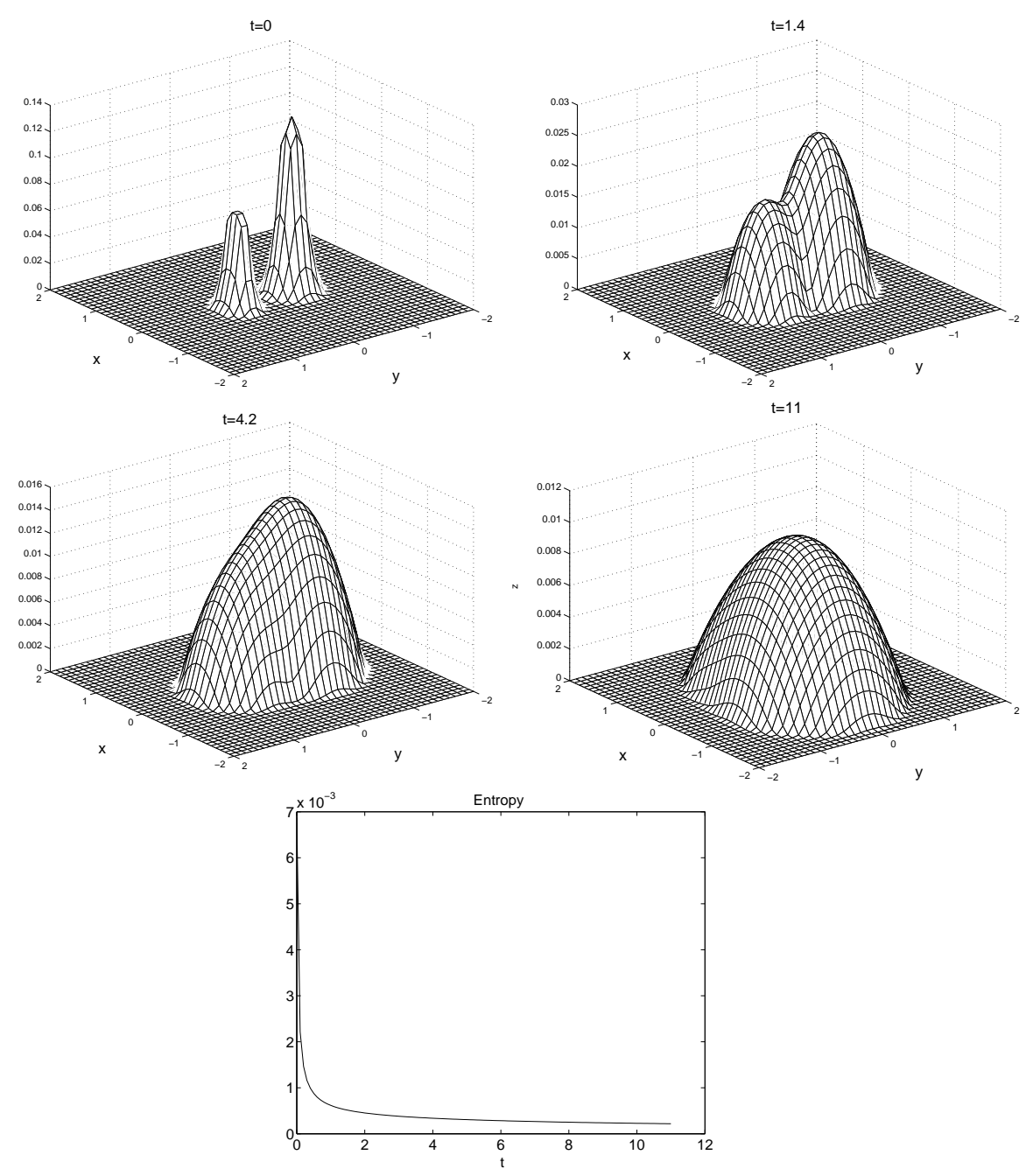

FIG. 2.2. Time evolution and entropy decay in time of the two dimensional nonlinear diffusion equation (2.6) in case $m=3$.

2.2. Nonlinear Fokker-Planck equation. This part of the paper is devoted to the investigation of problem $(2.1)$ in case $f(u):=u^{m}, m>1$ and $V(x):=\frac{1}{2} x^{2}$. It is well known that a standard central finite differences fully discretized implicit Euler scheme for (2.1) does not give nice results. This is due to the fact that if $\left|V_{x}\right|$ assumes large values where the function $u$ is small, the drift term $u V_{x}$ becomes predominant with respect to the diffusion term $f(u)_{x x}$, and this will cause undesired oscillations and large negative values in the solution (see Figure 2.3).

Therefore we follow the same scheme as in [24], used for a numerical approximation of the one dimensional transient drift-diffusion model for a bipolar semiconductor.

We briefly recall the most important steps. For the spatial discretization, we 


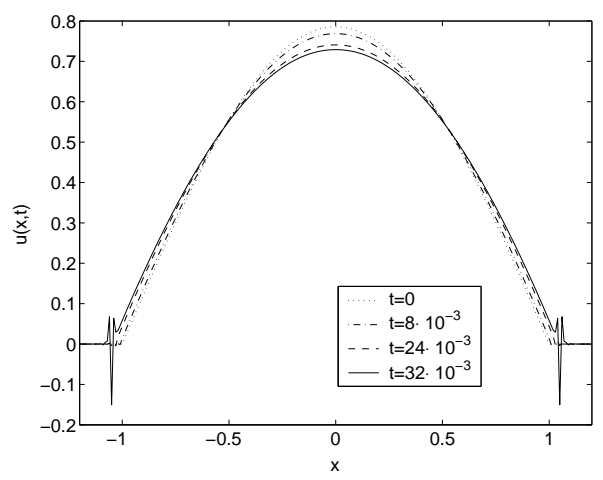

FIG. 2.3. Numerical results for (2.1) with central finite differences.

make use of a mixed exponential fitting method. The main idea is to linearize at each time step the current of the equation assuming $f^{\prime}\left(u\left(x, t_{k}\right)\right) \sim f^{\prime}{ }_{k}(x)$ is already known and rewrite the current term $J(x, t):=-\left(u(x, t) V_{x}(x)+f^{\prime}{ }_{k}(x) u_{x}(x, t)\right)$ into an equation for the new variable $z:=u e^{-V}$ in each spatial cell.

Let $x_{i}=i \Delta x$, where $i=1, \ldots, M$ and $\Delta x>0, I_{i}:=\left(x_{i-1}, x_{i}\right]$ and $J_{k}(i), u_{k}(i)$ and $V(i)$ denote the approximations at $x_{i}$ and at time $t_{k}:=k \Delta t$. The method can be summarized in two main steps: approximation of the diffusion term and change of variables.

It physically makes sense to expect that, if the current density $\tilde{J}_{k}$ in the interval $I_{i}$ is positive, the flow is moving to the right direction and then the density evaluated at the left $u_{k}(i-1)$ can be taken for the approximation of the coefficient of the diffusion term. More precisely we define

$$
f^{\prime}{ }_{k}(i):= \begin{cases}f^{\prime}\left(u_{k}(i-1)\right) & \text { if } \tilde{J}_{k}(i)>0 \\ f^{\prime}\left(u_{k}(i)\right) & \text { if } \tilde{J}_{k}(i) \leq 0 .\end{cases}
$$

We need an approximated value of the current $\tilde{J}_{k}(i)$ in $I_{i}$; for this we take

$$
\tilde{J}_{k}(i):= \begin{cases}0 & \text { if } u_{k}(i)=u_{k}(i-1)=0, \\ \frac{-1}{\Delta x}\left[\left(\phi^{\prime}\left(u_{k}(i)\right)-\phi^{\prime}\left(u_{k}(i-1)\right)\right)+(V(i)-V(i-1))\right] & \text { else. }\end{cases}
$$

We define now a new variable

$$
z_{k}:=u_{k} \exp \left(V / f^{\prime}{ }_{k}(i)\right) \text { in } I_{i}
$$

Then the expression of the current on the interval $I_{i}$ becomes

$$
\left.J_{k} \simeq-\left({f^{\prime}}_{k}(i) \exp \left(-V / f^{\prime}{ }_{k}(i)\right) z_{k, x}\right)\right),
$$

and equation (2.8) can be rewritten as

$$
\begin{array}{r}
\frac{1}{{f^{\prime}}(i)} \exp \left(V / f^{\prime}{ }_{k}(i)\right) J_{k}+z_{k, x}=0 \text { in } I_{i}, \\
\left(J_{k}\right)_{x}=-\frac{1}{\Delta t}\left(u_{k+1}-u_{k}\right) \text { in } I_{i} .
\end{array}
$$


We approximate now $J_{k}$ and $z_{k}$ as follows

$$
\begin{aligned}
& J_{k} \in X_{1}:=\left\{\varphi \in L^{2}(\Omega) \mid \varphi(x)=a_{i} x+b_{i}, x \in I_{i}, i=1, \ldots, M\right\}, \\
& z_{k} \in X_{0}:=\left\{\phi \in L^{2}(\Omega) \mid \phi \text { const. in } I_{i}, i=1, \ldots, M\right\},
\end{aligned}
$$

and taking $\tau \in X_{1}$ and $\phi \in X_{0}$ as test functions fot the above equations, the discrete system becomes

$$
\begin{aligned}
& \sum_{i=1}^{M}\left(\int_{I_{i}} \frac{1}{f^{\prime}{ }_{k}(i)} \exp \left(V / f^{\prime}{ }_{k}(i)\right) J_{k} \tau d x-\int_{I_{i}} z_{k} \tau_{x} d x+\left[u_{k} \exp \left(V / f^{\prime}{ }_{k}(i)\right) \tau\right]_{x_{i-1}}^{x_{i}}\right)=0, \\
& \sum_{i=1}^{M}\left(\int_{I_{i}}\left(J_{k}\right)_{x} \phi+\int_{I_{i}} \frac{1}{\Delta t}\left(u_{k+1}-u_{k}\right) \phi\right)=0 .
\end{aligned}
$$

We have now to approximate the last integrals. We choose $\phi=1$ in $I_{i}$ and $\phi=0$ elsewhere as test function, getting in this way

$$
J_{k}(i)-J_{k}(i-1)=-\frac{1}{\Delta t} \int_{I_{i}}\left(u_{k+1}-u_{k}\right) .
$$

The last integral is approximated as follows

$$
\int_{I_{i}}\left(u_{k+1}-u_{k}\right)=\Delta x\left(u_{k+1}(i-1)-u_{k}(i-1)\right) .
$$

It remains to compute $J_{k}$; first we approximate $J_{k}(x)=J_{k}(i)$ if $x \in I_{i}$, then taking $\tau=1$ in $I_{i}$ and $\tau=0$ elsewhere as test function, it holds

$$
\int_{I_{i}} \frac{1}{f_{k}^{\prime}(i)} \exp \left(V / f_{k}^{\prime}(i)\right) J_{k}(i) d x=-\left[u_{k} \exp \left(V / f^{\prime}{ }_{k}(i)\right)\right]_{x_{i-1}}^{x_{i}},
$$

which implies

$$
\begin{aligned}
J_{k}(i)= & -\left(\frac{V(i)-V(i-1)}{2} \operatorname{coth} \frac{V(i)-V(i-1)}{2 f_{k}^{\prime}(i)}\right) \frac{u_{k}(i)-u_{k}(i-1)}{\Delta x} \\
& -\frac{u_{k}(i)+u_{k}(i-1)}{2} \frac{V(i)-V(i-1)}{\Delta x} .
\end{aligned}
$$

This approximation for the flux is used in combination with an explicit Euler method

$$
\frac{u_{k+1}(i)-u_{k}(i)}{\Delta t}=-\frac{1}{\Delta x}\left(J_{k}(i+1)-J_{k}(i)\right) .
$$

Since the approximation for the flux is conservative, it is clear that the $L^{1}$-norm of the solution will be preserved at the fully discrete level.

Figure 2.4 shows the evolution in time of $(2.1)$ with $f(u)=u^{2}$. In this case the stationary-state of the Cauchy problem with the same mass as the initial data

$$
u_{I}(x)= \begin{cases}\frac{\pi}{4} \cos \left(\frac{\pi}{2} x\right) & \text { if }-1 \leq x \leq 1 \\ 0 & \text { otherwise }\end{cases}
$$

is given by $u_{\infty}(x)=\left(9^{1 / 3}-x^{2}\right)_{+}^{2} / 16$. Figure $2.4(\mathrm{~b})$ shows that the relative entropy (2.4), decays exponentially fast with rate -2 . 
(a)

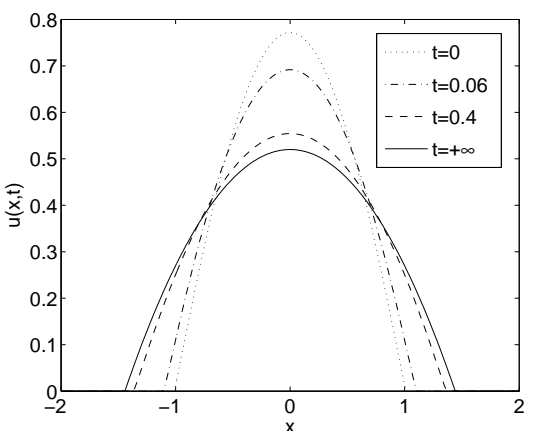

(b)

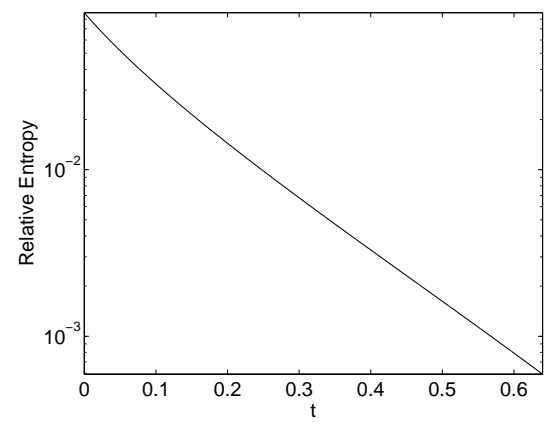

FIG. 2.4. Numerical results and entropy decay for (2.1) with initial condition (2.18) in the case $f(u)=u^{2}$ : (a) time evolution of $u(x, t)$, (b) logarithmic plot of the time evolution of $E(u)-E\left(u_{\infty}\right)$.

(a)

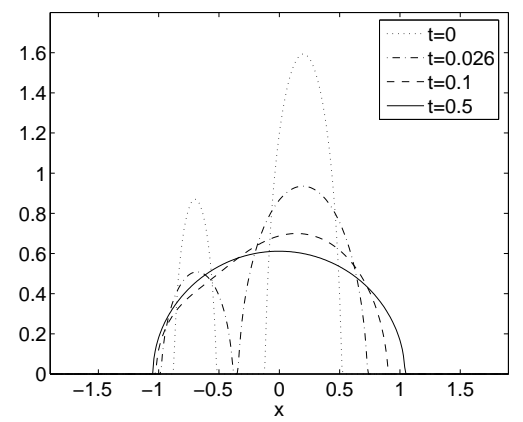

(b)

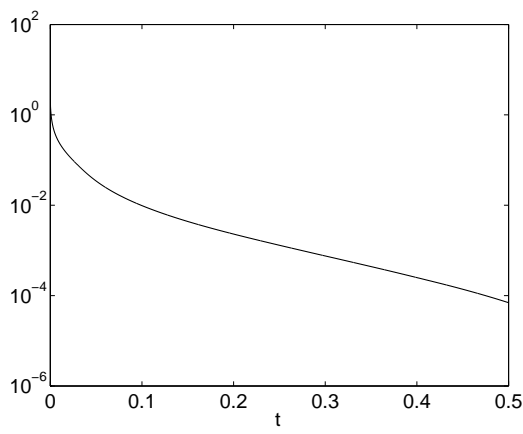

FIG. 2.5. Numerical results and entropy decay for (2.1) with initial condition (2.19) in case $f(u)=u^{3}$ : (a) time Evolution of $u(x, t)$, (b) logarithmic plot of the time evolution of $E(u)-E\left(u_{\infty}\right)$.

The numerical solution of (2.1) with initial condition

$$
u_{I}(x)= \begin{cases}-\frac{13}{3} x^{2}+\frac{5}{3} x & \text { if }-0.8 \leq x \leq-0.5 \\ -10 x^{2}-14 x-4.8 & \text { if }-0.1 \leq x \leq 0.5 \\ 0 & \text { otherwise }\end{cases}
$$

in the case $f(u)=u^{3}$ is showed in Figure 2.5. In this case, the convergence in time to a Barenblatt-type function is clear from the subplot 2.5(a). The relative entropy $E(u)-E\left(u_{\infty}\right)$ decreases numerically with constant rate -2 after an initial time interval, in which the decay is faster (Figure 2.5(b)). This behavior has been recently theoretically proven [13].

\section{Evolution of the 1-D Wasserstein distances}

In this section we analyze the Cauchy problem for general nonlinear diffusion equations (2.6) in one space dimension, i.e.

$$
\left\{\begin{array}{l}
v_{t}=f(v)_{x x}, \\
v(x, 0)=v_{I}(x),
\end{array}\right.
$$


where the initial datum $v_{I}$ is taken in $L_{+}^{1}(\mathbb{R})$. We require the nonlinearity function $f$ to satisfy the conditions as in Section 1 and moreover, $f$ satisfies the additional assumption

(F3) $f(v)=v^{m}+\Psi(v), m>1$, where $\Psi(v)=O\left(v^{n}\right)$ as $u \rightarrow 0$, for some $n>m$, $\Psi \in C^{1}((0,+\infty))$.

No condition on the growth of $\Psi$ at infinity is needed apart from $f$ satisfying the conditions of Section 1. This is due to the diffusion character of the equation. More precisely, values of the solution will tend pointwise to zero due to the $L^{1}-L^{\infty}$ effect and thus, the only important values of $\Psi$ affecting the long time behavior of the solutions are the values close to 0 . We perform the standard time dependent scaling

$$
\begin{array}{lll}
v(x, t)=R(t)^{-\frac{1}{\lambda}} w(y, s), & y=R(t)^{-\frac{1}{\lambda}}, & s=\frac{1}{\lambda} \log R(t), \\
R(t)=(1+\lambda t), & \lambda=m+1, &
\end{array}
$$

which turns (3.1) into

$$
\left\{\begin{array}{l}
w_{s}=\left(y w+e^{m s} f\left(e^{-s} w\right)_{y}\right)_{y}, \\
w(y, 0)=w_{I}(y)=v_{I}(y) .
\end{array}\right.
$$

In the sequel we shall assume for simplicity that $\int_{-\infty}^{+\infty} v_{I}(x) d x=1$. Our aim is to study, for any $p \geq 1$, the dynamic induced by (3.3) on the metric space

$$
\mathcal{M}_{2 p}=\left\{U(\cdot) \in L_{+}^{1}(\mathbb{R}), \int_{-\infty}^{+\infty}|x|^{2 p} U(x) d x<\infty\right\}
$$

endowed with the $2 p$-Wasserstein distance

$$
W_{2 p}\left(U_{1}, U_{2}\right)=\inf \left[\int_{-\infty}^{+\infty}|x-T(x)|^{2 p} U_{1}(x) d x\right]^{\frac{1}{2 p}},
$$

where the infimum is taken over the admissible maps $T: \mathbb{R} \rightarrow \mathbb{R}$ such that

$$
\int_{-\infty}^{+\infty} \psi(x) U_{2}(x) d x=\int_{-\infty}^{+\infty} \psi(T(x)) U_{1}(x) d x, \text { for all } \psi \in C^{0}(\mathbb{R}) .
$$

In one space dimension, the infimum in (3.4) is achieved and the optimal map $T^{*}$ can be expressed in a very simple way. Given two probability densities $U_{1}, U_{2} \in \mathcal{M}_{2 p}$, we define the distribution functions

$$
F(x)=\int_{-\infty}^{x} U_{1}(y) d y, \quad G(x)=\int_{-\infty}^{x} U_{2}(y) d y,
$$

and their pseudo-inverses $F^{-1}, G^{-1}:[0,1] \rightarrow \mathbb{R}$

$$
F^{-1}(\rho)=\inf \{\omega: F(\omega)>\rho\} \quad G^{-1}(\rho)=\inf \{\omega: G(\omega)>\rho\}
$$

(eventually $F^{-1}$ and $G^{-1}$ may attain the values $\pm \infty$ at $\rho=0$ or at $\rho=1$ ). Then, it can be proven by direct computation that the optimal map $T^{*}$ between the measures $U_{1} d x$ and $U_{2} d x$ is the unique admissible map $T^{*}=G^{-1} \circ F$. Hence, by writing down (3.4) in terms of $T^{*}$, after a change of variable, we get

$$
W_{2 p}\left(U_{1}, U_{2}\right)=\left[\int_{0}^{1}\left|F^{-1}(\rho)-G^{-1}(\rho)\right|^{2 p} d \rho\right]^{\frac{1}{2 p}} .
$$


We refer to [39] for a detailed explanation of these topics.

Thanks to the monotonicity of $W_{2 p}\left(U_{1}, U_{2}\right)$ with respect to the index $p$, one can eventually send $p \rightarrow \infty$ to obtain

$$
W_{\infty}\left(U_{1}, U_{2}\right)=\sup _{\rho \in(0,1)}\left|F^{-1}(\rho)-G^{-1}(\rho)\right| .
$$

It can easily be seen that, whenever $U_{1}$ and $U_{2}$ have compact support, the quantity $W_{\infty}\left(U_{1}, U_{2}\right)$ provides an estimate of the 'relative' speed of propagation of the supports of $U_{1}$ and $U_{2}$ respectively. More precisely, it holds (see $[19,14]$ )

$$
\begin{aligned}
& \left|\inf \left\{\operatorname{supp} U_{1}\right\}-\inf \left\{\operatorname{supp} U_{2}\right\}\right| \leq W_{\infty}\left(U_{1}, U_{2}\right) \\
& \left|\sup \left\{\operatorname{supp} U_{1}\right\}-\sup \left\{\operatorname{supp} U_{2}\right\}\right| \leq W_{\infty}\left(U_{1}, U_{2}\right) .
\end{aligned}
$$

Moreover, whenever $U_{1}$ has compact support, then $W_{\infty}\left(U_{1}, U_{2}\right)$ is finite if and only if $U_{2}$ has compact support.

Let us consider for the moment the case of a homogeneous nonlinearity $f(u)=u^{m}$, which corresponds to the porous medium equation in (3.1). We are interested in computing the Wasserstein distance between any nonnegative $w$ solution with unit mass of the rescaled equation

$$
w_{s}=\left(y w+\left(w^{m}\right)_{y}\right)_{y},
$$

and the corresponding self-similar Barenblatt profile $w_{\infty}$ with unit mass defined in $(2.7)$ written in similarity variables $(y, s)$, i.e. the stationary profile

$$
w_{\infty}(y)=\left(C-\frac{m-1}{2 m}|y|^{2}\right)_{+}^{\frac{1}{m-1}},
$$

for some constant $C$. In order to study the evolution of such a quantity, we set

$$
F(y, s)=\int_{-\infty}^{y} w(z, s) d z, \quad G(y, s)=\int_{-\infty}^{y} w_{\infty}(z) d z .
$$

Let $F^{-1}, G^{-1}:(0,1) \rightarrow \mathbb{R}$ be the pseudo-inverses of $F$ and $G$ respectively. Then, $F^{-1}$ satisfies the following equation (see $[19,14]$ )

$$
\frac{\partial F^{-1}}{\partial s}=-F^{-1}-\frac{\partial}{\partial \rho}\left[\left(\frac{\partial F^{-1}}{\partial \rho}\right)^{-m}\right],
$$

while $G^{-1}$ satisfies

$$
G^{-1}+\frac{\partial}{\partial \rho}\left[\left(\frac{\partial G^{-1}}{\partial \rho}\right)^{-m}\right]=0
$$

Equations (3.8) and (3.9) provide a direct computation of the $L^{2 p}$-norms of the difference $F^{-1}-G^{-1}$, and therefore, an estimate of the $2 p$-Wasserstein distance between $w$ and $w_{\infty}$, as shown in [14]. As we will see later on, the diffusion term

$$
-\frac{\partial}{\partial \rho}\left[\left(\frac{\partial F^{-1}}{\partial \rho}\right)^{-m}\right],
$$


in (3.8) is dissipative, while the term $-F^{-1}$ provides an exponential decay. More precisely, we have

$$
W_{2 p}\left(w(s), w_{\infty}\right) \leq e^{-s} W_{2 p}\left(w_{I}, w_{\infty}\right) .
$$

In the original variables, we have the following contraction property

$$
W_{2 p}\left(v(t), B\left(x, t+\lambda^{-1}\right)\right) \leq W_{2 p}\left(v_{I}, B\left(x, \lambda^{-1}\right)\right),
$$

where $B\left(x, t+\lambda^{-1}\right)$ is the Barenblatt profile corresponding to $w_{\infty}$ in the original variables. Sending $p \rightarrow \infty$ yields contraction of the $W_{\infty}$ and, thanks to (3.6),

$$
\begin{aligned}
& \left|\inf \{\operatorname{supp} v(t)\}-\inf \left\{\operatorname{supp} B\left(x, t+\lambda^{-1}\right)\right\}\right| \leq W_{\infty}\left(v_{I}, B\left(x, \lambda^{-1}\right)\right), \\
& \left|\sup \{\operatorname{supp} v(t)\}-\sup \left\{\operatorname{supp} B\left(x, t+\lambda^{-1}\right)\right\}\right| \leq W_{\infty}\left(v_{I}, B\left(x, \lambda^{-1}\right)\right) .
\end{aligned}
$$

In fact, the last lines can even be improved to show the finite speed of propagation without assuming it from the beginning. Since the $W_{\infty}$-distance is contractive and $B\left(x, t+\lambda^{-1}\right)$ has compact support at any time $t \geq 0$, taking compactly supported initial data $v_{I}$, the quantity $W_{\infty}\left(v(t), B\left(x, t+\lambda^{-1}\right)\right)$ stays finite and immediately it follows that $v(t)$ has compact support. In [14] the above arguments are performed rigorously, by means of an approximation of the original Cauchy problem by an initial boundary value problem on a closed interval eventually tending to the whole real line. Our purpose is to generalize the previous approach to the perturbed case $f(u)=$ $u^{m}+\Psi(u)$ according to assumption (F3). Before proceeding further, let us remark that these results were already proven in [34] by comparison arguments. The novelty here is the proof by completely different arguments that might be generalized to larger dimensions at least asymptotically.

We shall perform the estimate of the Wasserstein distances directly on the solutions of the Cauchy problem. The computations below can be made rigorous in a similar fashion as in [14]. To heuristically justify our procedure, let us turn back (3.3). By formally letting $s$ tend to infinity, we deduce that the evolution of the solution $w(s)$ for large $s$ is governed by the power term $w^{m}$. Therefore, we expect that the Wasserstein distance between the rescaled solution $w(\cdot, s)$ of $(3.3)$ and the corresponding stationary Barenblatt profile $w_{\infty}$ defined in (3.7) tends to zero as $s$ goes to infinity with the same exponential rate as for the porous medium equation. Given a solution $w$ to (3.3) and given the stationary Barenblatt profile with unit mass $w_{\infty}$ corresponding to the power nonlinearity $u^{m}$, we denote once again

$$
F(y, s)=\int_{-\infty}^{y} w(z, s) d z, \quad G(y, s)=\int_{-\infty}^{y} w_{\infty}(z) d z .
$$

The two corresponding pseudo-inverses $F^{-1}$ and $G^{-1}$ satisfy

$$
\begin{aligned}
& \frac{\partial F^{-1}}{\partial s}=-F^{-1}-\frac{\partial}{\partial \rho}\left\{\left[\frac{\partial F^{-1}}{\partial \rho}\right]^{-m}+e^{m s} \Psi\left(e^{-s}\left(\frac{\partial F^{-1}}{\partial \rho}\right)^{-1}\right)\right\}, \\
& G^{-1}+\frac{\partial}{\partial \rho}\left[\left(\frac{\partial G^{-1}}{\partial \rho}\right)^{-m}\right]=0 .
\end{aligned}
$$

We next state our result concerning Wasserstein distances.

Theorem 3.1. Let $f(v)$ satisfy conditions (F1)-(F3) stated before. 
(a) Let $w(y, s)$ be the solution to (3.3) with $v_{I} \in L_{+}^{1}(\mathbb{R})$ having unit mass and finite second moment. Then, for any $p \geq 1$,

$$
W_{2 p}\left(w(s), w_{\infty}\right)=\left[\int_{0}^{1}\left|F^{-1}(\rho)-G^{-1}(\rho)\right|^{2 p} d \rho\right]^{\frac{1}{2 p}} \leq C e^{-s},
$$

holds, where $C=C_{0}+W_{2 p}\left(v_{I}, w_{\infty}\right)$ and $C_{0}$ depends only on $f$.

(b) Let $w(y, s)$ be the solution to (3.3) with $v_{I} \in L_{+}^{1}(\mathbb{R})$ having unit mass and compact support. Then

$$
W_{\infty}\left(w(s), w_{\infty}\right) \leq C e^{-s},
$$

where $C=C_{0}+W_{\infty}\left(v_{I}, w_{\infty}\right)$ and $C_{0}$ depends only on $f$.

In original variables (3.1), part (b) of the previous theorem provides a result concerning the expansion rate of the support of any solution $v(x, t)$ having compactly supported initial datum $v_{I}$. Indeed, since the support of the Barenblatt profile is a ball of radius $C\left(t+\lambda^{-1}\right)^{\frac{1}{m+1}}$ for some fixed constant $C>0$, we easily obtain the following consequence.

Corollary 3.2. Let $v(x, t)$ be solution to (3.1) with $v_{I} \in L_{+}^{1}(\mathbb{R})$ having compact support, let $B\left(x, t+\lambda^{-1}\right)$ be the Barenblatt profile with same mass. Then, there exist a fixed constant $C$ such that

$$
\begin{aligned}
& \left|\inf \{\operatorname{supp} v(t)\}-\inf \left\{\operatorname{supp} B\left(x, t+\lambda^{-1}\right)\right\}\right| \leq C, \\
& \left|\sup \{\operatorname{supp} v(t)\}-\sup \left\{\operatorname{supp} B\left(x, t+\lambda^{-1}\right)\right\}\right| \leq C .
\end{aligned}
$$

REMARK 3.3. We found a lot of references in the literature concerning the finite speed of propagation property in slow diffusion equations (see $[26,25]$ for the general nonlinear case). Most of them are based on heavy analytic tools. Our result is more complete in the general nonlinear case, and covers a wider class of nonlinearities. Moreover, this technique applies to this problem in a very natural way.

Proof of Theorem 3.1. To perform the proof of Theorem 3.1, we compute the evolution of $W_{2 p}\left(w(s), w_{\infty}\right)$ by means of the one-dimensional representation formula (3.5). The calculations below are formal, in the sense that we should need the pseudo-inverse function $F^{-1}$ to be smooth enough. We observe that this occurs when the initial datum $v_{I}$ is supported on a interval. We could make this argument rigorous by means of standard approximation tools (see [14]). We skip these details and suppose that $F^{-1}$ is smooth.

Moreover, we need to know a priori that the speed of propagation of the support of the solution is finite. This property, which actually characterizes slow diffusion equations, was proved by Kalashnikov, Oleinik and Yiu-Lin (see [25] and the references therein). In fact, a refinement of the argument leads to proof that the speed of propagation is finite without assuming it, by showing the control on $W_{\infty}$-distance by approximations. Using the notations of the previous subsection, thanks to (3.11) and 
after integration by parts, we have

$$
\begin{aligned}
\frac{d}{d s} \int_{0}^{1}\left[F^{-1}-G^{-1}\right]^{2 p} d \rho=2 p \int_{0}^{1}\left[F^{-1}-G^{-1}\right]^{2 p-1} \frac{\partial}{\partial s} F^{-1}(\rho, s) d \rho \\
=2 p \int_{0}^{1}\left[F^{-1}-G^{-1}\right]^{2 p-1}\left[-F^{-1}-\frac{\partial}{\partial \rho}\left(\left(\frac{\partial F^{-1}}{\partial \rho}\right)^{-m}\right.\right. \\
\left.\left.\quad+e^{m s} \Psi\left(e^{-s}\left(\frac{\partial F^{-1}}{\partial \rho}\right)^{-1}\right)\right)+G^{-1}+\frac{\partial}{\partial \rho}\left(\left(\frac{\partial G^{-1}}{\partial \rho}\right)^{-m}\right)\right] d \rho \\
=-2 p \int_{0}^{1}\left[F^{-1}-G^{-1}\right]^{2 p} d \rho-2 p(2 p-1) \int_{0}^{1}\left[F^{-1}-G^{-1}\right]^{2 p-2}\left(\frac{\partial F^{-1}}{\partial \rho}-\frac{\partial G^{-1}}{\partial \rho}\right) \\
\quad \times e^{m s}\left[f\left(e^{-s}\left(\frac{\partial F^{-1}}{\partial \rho}\right)^{-1}\right)-f\left(e^{-s}\left(\frac{\partial G^{-1}}{\partial \rho}\right)^{-1}\right)\right] d \rho \\
\quad-2 p \int_{0}^{1}\left[F^{-1}-G^{-1}\right]^{2 p-1} \frac{\partial}{\partial \rho}\left(e^{m s} \Psi\left(e^{-s}\left(\frac{\partial G^{-1}}{\partial \rho}, s\right)^{-1}\right)\right) d \rho .
\end{aligned}
$$

We observe that, due to the compact support of the solutions, the boundary term coming from integration by parts disappears (see $[19,14]$ ). In fact, this boundary term is given by

$$
\begin{aligned}
\sum_{i=0,1}(-1)^{i} 2 p\left[F^{-1}(i, s)\right. & \left.-G^{-1}(i)\right]^{2 p-1}\left[\left(\frac{\partial F^{-1}}{\partial \rho}\right)^{-m}(i, s)\right. \\
+ & \left.e^{m s} \Psi\left(e^{-s}\left(\frac{\partial F^{-1}}{\partial \rho}\right)^{-1}\right)(i, s)-\left(\frac{\partial G^{-1}}{\partial \rho}\right)^{-m}(i, s)\right]
\end{aligned}
$$

The first bracket is bounded at any $s$ because of the finite speed of propagation property of the solutions. The second bracket is a sum of positive powers of the solution $w$ and of the Barenblatt function $w_{\infty}$ evaluated at the boundary of their support respectively. Hence, this second bracket equals zero.

Now, since the function $f$ is increasing, the second integral at the end of (3.15) is nonnegative. This observation is the key point in this computation (see again [14]). In fact, thanks to this we can get rid of the nonlinear term, and we have only to estimate the term depending on the Barenblatt profile, which is known. Indeed, after some calculations in the very last term of (3.15), due to the equation satisfied by $G^{-1}$ in (3.11), we obtain the following inequality

$$
\begin{aligned}
& \frac{d}{d s} \int_{0}^{1}\left[F^{-1}-G^{-1}\right]^{2 p} d \rho \leq-2 p \int_{0}^{1}\left[F^{-1}-G^{-1}\right]^{2 p} d \rho \\
& \quad-\frac{2 p}{m} e^{(m-1) s} \int_{0}^{1}\left[F^{-1}-G^{-1}\right]^{2 p-1} G^{-1} \Psi^{\prime}\left(e^{-s}\left(\frac{\partial G^{-1}}{\partial \rho}\right)^{-1}\right)\left(\frac{\partial G^{-1}}{\partial \rho}\right)^{m-1} d \rho .
\end{aligned}
$$

We can assume that $\Psi(v)=v^{n} g(v)$, with $g^{\prime}(v)=O\left(v^{k}\right), k>-1$, as $v \rightarrow 0$. Then, it follows that $\Psi^{\prime}(v)=O\left(v^{n-1}\right)$, as $v \rightarrow 0$. Hence, by Hölder inequality, the last integral 
above can be estimated from above by the term

$$
p C\left(w_{\infty}\right) e^{-(n-m) s}\left(\int_{0}^{1}\left|F^{-1}-G^{-1}\right|^{2 p-1} d \rho\right)^{\frac{2 p-1}{2 p}},
$$

where the constant $C\left(w_{\infty}\right)$ is given by

$$
C\left(w_{\infty}\right)=\left\|w_{\infty}\right\|_{L^{\infty}(\mathbb{R})} \max \left\{\left|\inf \left\{\operatorname{supp} w_{\infty}\right\}\right|,\left|\sup \left\{\operatorname{supp} w_{\infty}\right\}\right|\right\}
$$

that depends only on the mass and on the exponent $m$. We now apply the variation of constants formula in order to get the rate of convergence to zero of $W_{p}\left(w(s), w_{\infty}\right)$. In order to perform this task, we set for simplicity

$$
\mathcal{X}_{p}(s)=\int_{0}^{1}\left[F^{-1}(\rho, s)-G^{-1}(\rho)\right]^{2 p} d \rho .
$$

Hereafter, $C$ denotes a fixed positive constant, independent on $p$ and $s$. So far we have proved that

$$
\frac{d}{d s} \mathcal{X}_{p}(s) \leq-2 p \mathcal{X}_{p}(s)+2 p C e^{-(n-m) s} \mathcal{X}_{p}(s)^{\frac{2 p-1}{2 p}} .
$$

By Young inequality we get

$$
\frac{d}{d s} \mathcal{X}_{p}(s) \leq-2 p\left(1-C e^{-(n-m) s}\right) \mathcal{X}_{p}(s)+C e^{-(n-m) s},
$$

and the variation of constants formula implies that

$$
\mathcal{X}_{p}(s) \leq\left(\mathcal{X}_{p}(0)+C\right) e^{-\min \{2 p,(n-m)\} s} .
$$

In case that $n-m<2 p$, the exponential rate of convergence in (3.17) can be improved iteratively by substituting the above inequality in the last addend of (3.16), until it reaches the value $e^{-2 p s}$. Obviously, the number of steps depends on $p$. We have thus proved (3.12). Inequality (3.13) easily follows by letting $p \rightarrow \infty$.

Figure 3.1 shows the evolution of the $p$-Wasserstein distance defined in (3.5) for several values of $p$ between $v_{1}(x, t)$ and $v_{2}(x, t)$, solutions of (2.15) with initial conditions (2.16) and (2.18) respectively in case $f(v)=v^{2}$. The integral in (3.5) for the forthcoming tests is computed by numerical quadrature and we are using the fully discretized implicit Euler scheme described in Subsection 2.1.

It is interesting to observe that although the distance between the solutions is only known to be a contraction, this distance is in fact decaying quickly as $t \rightarrow \infty$. Let us point out that the two initial data have zero center of mass and therefore are well centered. In fact, in [12] we have shown that the Euclidean Wasserstein distance between the solutions when you fix the center of mass of the initial data decays. In the case of expansion rate of supports, this was already observed by J. L. Vázquez in [34].

\section{Intermediate asymptotics for general nonlinearities}

In this section we summarize a result contained in [11] concerning the long time behavior for a general nonlinear diffusion equation

$$
\frac{\partial v}{\partial t}=\Delta f(v), \quad x \in \mathbb{R}^{N}, \quad t>0,
$$

where $f$ satisfies the the following assumptions: 
(a)

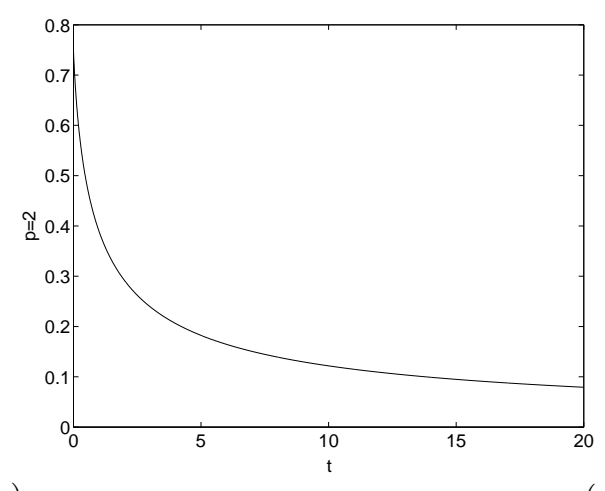

(b)

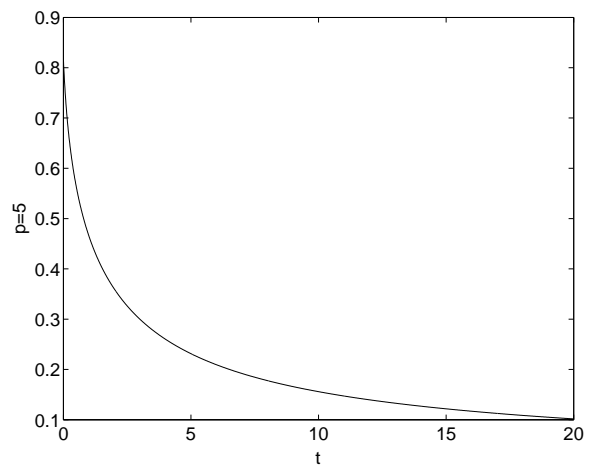

(c)

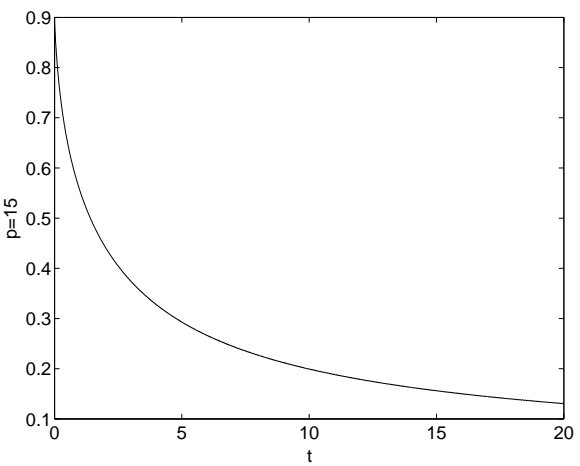

FIG. 3.1. $p$-Wasserstein distance $W_{p}\left(v_{1}, v_{2}\right)$.

(NL1) $f \in C[0,+\infty) \cap C^{1}(0,+\infty), f(0)=0$ and $f^{\prime}(v)>0$ for all $v>0$,

(NL2) $\exists C>0$ and $m>\frac{N-2}{N}$ such that $f^{\prime}(v) \geq C v^{m-1}$ for all $v>0$,

(NL3) $\frac{f(v)}{v^{1-1 / N}}$ is nondecreasing on $v \in(0, \infty)$.

Assumption (NL1) ensures that (4.1) is well-posed for any initial datum in $L_{+}^{1}\left(\mathbb{R}^{N}\right)$ (see $[6,38]$, see also $[9,7]$ for more qualitative properties of solutions). Under the additional assumption (NL2), (4.1) enjoys an $L^{1}-L^{\infty}$ regularizing property. Indeed, it is proved that the solution to (4.1) with initial datum in $L_{+}^{1}\left(\mathbb{R}^{N}\right)$ satisfies the following in time decay estimate (see [4] for the power law case, [38] for the general nonlinear case)

$$
\|v(\cdot, t)\|_{L^{\infty}\left(\mathbb{R}^{N}\right)} \leq C t^{-\frac{N}{\lambda}}\left\|v_{I}\right\|_{L^{1}\left(\mathbb{R}^{N}\right)}^{\frac{2}{\lambda}} .
$$

Assumption (NL3) implies that the entropy functional associated to (4.1) is displacement convex [28] and thus, the flow map of the nonlinear diffusion (4.1) is a nonexpansive contraction in time with respect to the euclidean Wasserstein distance $W_{2}$ in probability measures $[29,17,2]$. 
As previously observed, in the power law case $f(v)=v^{m}$ self-similar solutions of the form (2.7) can be seen as stationary profiles of (4.1) written in similarity variables, i.e., the nonlinear Fokker-Planck equation (2.1) with $V(x)=\frac{|x|^{2}}{2}$ and $f(u)=$ $u^{m}$. Moreover, relative entropy tools provide exponential convergence towards such stationary profiles. In case of a general nonhomogeneous nonlinearity $f(v)$ there are no time-dependent scalings which allow rewriting (4.1) in the form of (2.1); therefore it was an open problem how to detect any special solution as a reasonable candidate to be the universal asymptotic profile for (4.1).

We first define the "temperature" of a solution $v$ to (4.1) as its second moment, i.e.,

$$
\theta_{v}(t)=\int_{\mathbb{R}^{N}} \frac{|x|^{2}}{2} v(x, t) d x .
$$

Given a solution $v(t)$ to (4.1) we will study the long-time behavior of

$$
\theta_{v}(t)^{N / 2} v\left(\theta_{v}(t)^{1 / 2} x, t\right)
$$

Similar scalings have been used in the analysis of homogeneous cooling states in granular media equations (see, for instance, $[5,31]$ and the references therein). The nonlinear time dependent scaling (4.3) can be also seen as the projection of the solution $v(\cdot, t)$ onto the manifold of probability measures with unit temperature (see also [10])

$$
\mathcal{M}=\left\{\mu \in \mathcal{P}_{2}\left(\mathbb{R}^{N}\right), \frac{1}{2} \int_{\mathbb{R}^{N}}|x|^{2} d \mu(x)=1\right\} .
$$

In order to get the above scaling (4.3) well defined for all positive times $t$, we must require in addition the following natural assumption on the nonlinearity $f$, namely

(FT) for any solution $v(x, t)$ to (4.1), it holds,

$$
\int_{\mathbb{R}^{N}}|x|^{2} v(x, 0) d x<\infty, \Rightarrow \int_{\mathbb{R}^{N}}|x|^{2} v(x, t) d x<\infty \text { for all } t>0 .
$$

In [11] we prove that the above assumption is satisfied under reasonable requirements for the function $f$, also including fast diffusion ranges of nonlinearities.

For further reference, we state the following lemma, which ensures that $\theta_{v}(t)$ tends to infinity as $t \rightarrow+\infty$ (see [11] for the proof).

Lemma 4.1. Suppose that $f$ satisfies assumptions (NL1),(NL2) and (FT). It holds

$$
\theta(v)(t) \geq C_{0} t^{\frac{2}{d(m-1)+2}},
$$

where $C_{0}$ depends only on the mass of $v$.

Our main result is the following:

THEOREM 4.2 (Asymptotic profile for general nonlinear diffusions). Given $f$ verifying hypotheses (NL1)-(NL3) and $(\boldsymbol{F T})$, there exists $t_{*}>0$ and a one parameter curve of probability densities $v_{\infty}(t)$, with unit temperature defined for $t \geq t_{*}$ such that, for any solution of (4.1) with initial data $\left(1+|x|^{2}\right) v_{I} \in L_{+}^{1}\left(\mathbb{R}^{N}\right)$ of unit mass and temperature,

$$
W_{2}\left(\theta_{v}(t)^{N / 2} v\left(\theta_{v}(t)^{1 / 2} \cdot, t\right), v_{\infty}(t)\right) \longrightarrow 0 \quad \text { as } \quad t \rightarrow \infty .
$$


Moreover, the asymptotic profile $v_{\infty}(t)$ is characterized as the unique fixed point of the renormalized flow map $S(t)$

$$
S(t) v_{I}:=\theta_{v}(t)^{N / 2} v\left(\theta_{v}(t)^{1 / 2} \cdot, t\right)
$$

where $v(\cdot, t)$ is the solution to (4.1) with initial datum $v_{I}$.

The main ingredient in previous theorem is the proof of a contraction property for the maps $S(t), t \geq t_{*}>0$, obtained as compositions of the flow map for (4.1) and the projection of the solution onto the unit second moment manifold $\mathcal{M}$. In the case of the homogeneous nonlinearities $f(v)=v^{m}$ the asymptotic profile $v_{\infty}(t)$ is nothing else but the Barenblatt-Prattle solution at the time in which it has unit temperature (therefore is constant in time) [32]. Indeed, thanks to our point of view we can generalize the classical notions of self-similarity and source-type solution by means of the idea of invariance of the solution orbit after projection onto the subset $\mathcal{M}$. The proof of contraction of $S(t)$ with respect to the euclidean Wasserstein distance makes use of the $L^{1}-L^{\infty}$ regularizing effect, needed to control from below the behavior of the temperature of the solution as $t \rightarrow \infty$. Then, the proof is based on elementary properties of the euclidean Wasserstein distance, which allow to generalize our approach to more situations. Open problems are the eventual convergence of $v_{\infty}(t)$ to a unique limit point as $t \rightarrow \infty$, and convergence results in $L^{p}$ spaces for solutions of (4.1) to such a limit. We can provide an answer only in case $f(v)$ satisfies assumption (F3) of the previous section, i.e. when $f$ is a higher order perturbation of a power law $v^{m}$. In that case, we could prove convergence towards the corresponding Barenblatt profile (see [11]).

TheOREM 4.3 (Asymptotically homogeneous nonlinearities). Let $v(x, t)$ be the solution to (4.1) with nonlinearity $f$ satisfying hypotheses (NL1)-(NL3), (F3) and (FT) above with initial datum $v_{I} \in L_{+}^{1}\left(\mathbb{R}^{N}\right)$ such that

$$
\int_{\mathbb{R}^{N}}\left[v_{I}^{m}(x)+|x|^{2} v_{I}(x)+\phi\left(v_{I}(x)\right)\right] d x<+\infty .
$$

Let $B(|x|, t)$ be the Barenblatt self-similar function with the same mass as $v_{I}$ corresponding to the exponent $m$. Then, the following estimate holds for all $t \geq 0$

$$
\|v(\cdot, t)-B(|\cdot|, t)\|_{L^{1}\left(\mathbb{R}^{N}\right)} \leq C(t+1)^{-\frac{\delta}{\lambda \alpha}},
$$

where

$$
\lambda=N(m-1)+2, \quad \alpha=\left\{\begin{array}{ll}
2 & \text { if } m \leq 2 \\
m & \text { if } m \geq 2,
\end{array} \quad \delta=\min \{2, N n\}\right.
$$

$n$ is given by condition (F3) and $C$ depends only on the initial datum $v_{I}$.

4.1. Numerical results. The aim of this section is to compute numerically the asymptotic profiles $v_{\infty}(t)$ and try to clarify if that profile has a unique limit as $t \rightarrow \infty$. All the results in this section are obtained by applying a simple fixed point iteration on the maps $S(t)$ for several values of $t$ chosen uniformly over a time interval $\left[T_{1}, T_{2}\right]$. Therefore, one uses the fully discretized Euler implicit scheme introduced in Subsection 2.1 to compute the solution to (4.1) for each value of the iteration till eventual convergence of the fixed point iteration up to a fixed tolerance. Let us finally remark that all theoretical results were written by projecting the solution onto the 
manifold of measures with unit second moment, of course, everything can be generalized by projecting onto the manifold with an arbitrary fixed temperature and we will do so for numerical convenience.

Figure (4.1) is a benchmark for the scheme since we know theoretically that the asymptotic profile of the problem $v_{t}=\left(v^{2}\right)_{x x}$ is the Barenblatt $(2.7) B\left(x, t^{*}\right)$ with $C$ fixed by conservation of mass $\left\|v_{I}(\cdot)\right\|_{L^{1}(\mathbb{R})}=\left\|B\left(\cdot, t^{*}\right)\right\|_{L^{1}(\mathbb{R})}$ and $t^{*}$ fixed by the initial second moment:

$$
\int_{\mathbb{R}} x^{2} v_{I}(x) d x=\int_{\mathbb{R}} x^{2} B\left(|x|, t^{*}\right) d x
$$

We take as initial data $v_{I}$ defined by $(2.16)$ and we have plotted all the $v_{\infty}(t)$ fixed points onto a time interval $[5,14]$ that match each other and $B\left(x, t^{*}\right)$ as expected.

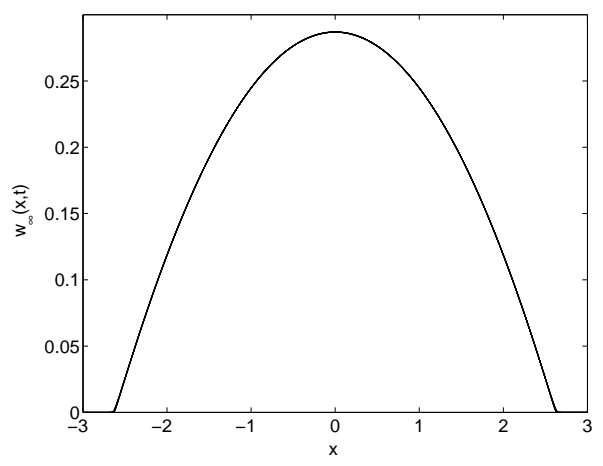

FIG. 4.1. Asymptotic profile of (4.1) with $f(v)=m^{2}$.

Figure (4.2) shows the asymptotic profile for (4.1) with $f(v)=v^{2}+v^{3}$ and taking as initial datum $v_{I}(x)$ defined by $(2.16)$ to fix the mass and the temperature of the asymptotic profile like before and to start the fixed point iteration. Since this case is a perturbation of the homogeneous equation with $f(v)=v^{2}$ in the sense of hypothesis (F3), then the asymptotic profile should approach the Barenblatt corresponding to $f(v)=v^{2}$ with initial mass and time fixed by the initial second moment. Results shown in this figure support this fact since the computed values of the asymptotic profile $v_{\infty}(t)$ converges increasingly to the expected limiting Barenblatt (dotted line in subplot $(4.3)(\mathrm{a})$ and (b)).

The asymptotic profile of (4.1) with

$$
f(v)=\frac{v^{2}}{v^{2}+0.5(1-v)^{2}},
$$

is plotted in Figure (4.3). The initial datum $v_{I}$ is defined by (2.16). Note that $f$ defined by (4.5) satisfies (F2) for $0 \leq v \leq 1$, then this case is a perturbation of (2.6) with $f(v)=v^{2}$. The approach of the asymptotic profile to the Barenblatt corresponding to $f(v)=v^{2}$ with the initial mass and time fixed by the initial second moment (dotted line in subplot (4.3)(a) and (b)) is clear from the subplot (4.3)(b).

Finally in Figure (4.4), we show the asymptotic profile for (4.1), where $f$ is defined as the primitive of

$$
f^{\prime}(v):=v\left(1+2 \sin ^{2}\left(\frac{10}{v}\right)\right)
$$


(a)
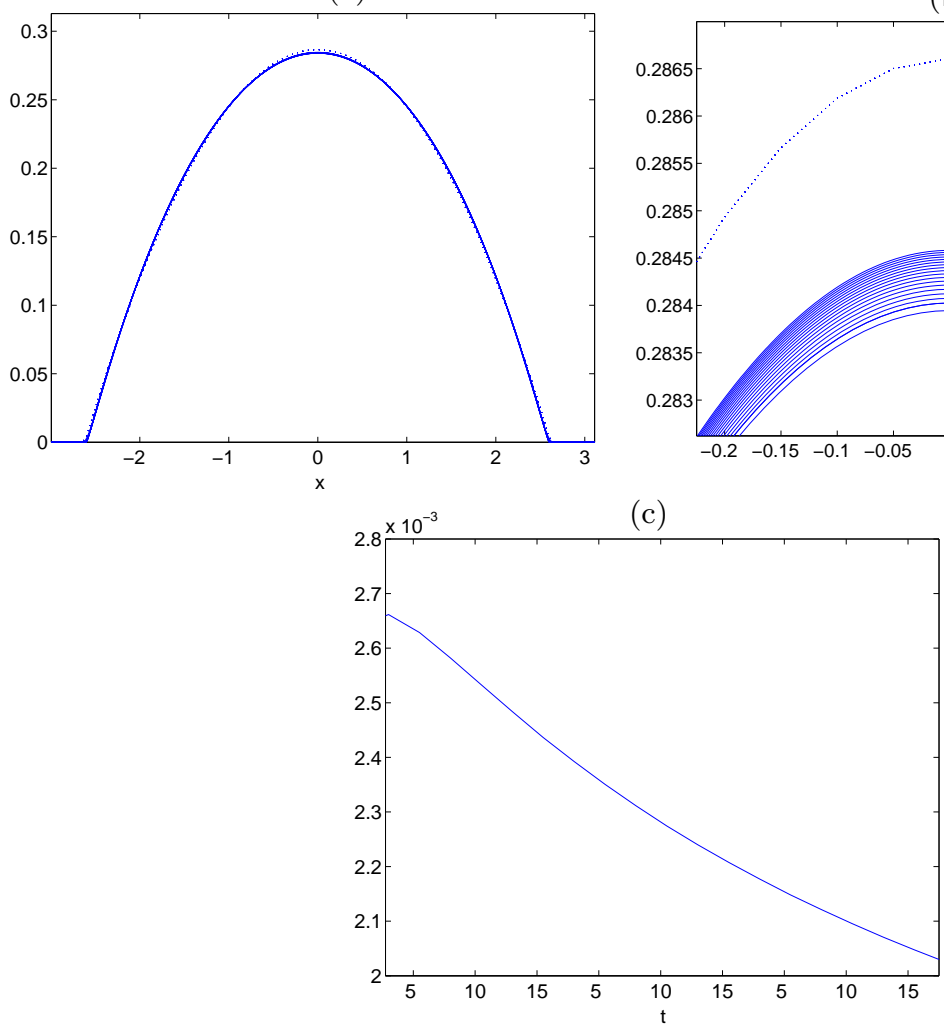

(b)

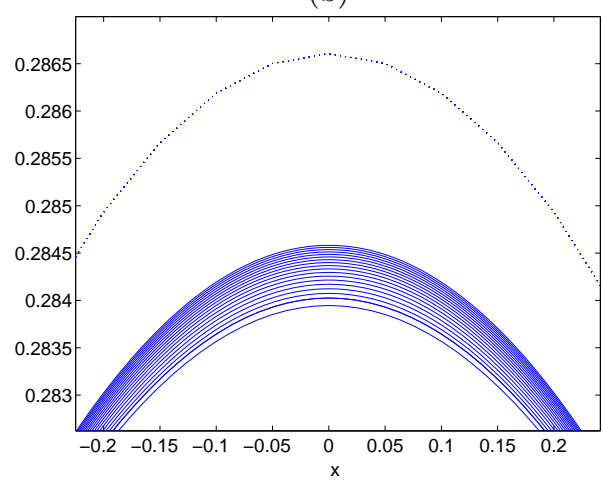

$$
\text { (1) }
$$

$+2$
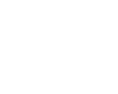

FIG. 4.2. (a) Asymptotic profile of (4.1) with $f(v)=v^{2}+v^{3}$, (b) Zoom of subplot (a) around $x=0,(c)\left|v_{\infty}(x=0, t)-B(x=0)\right|$.

This case is chosen in such a way that the derivative of the nonlinearity oscillates near zero. We observe that the computed values of the asymptotic profiles oscillate and at least up to time 17 they do not stabilize in time. Recently, we have given [20] a construction of nonlinearities allowing oscillations as $t \rightarrow \infty$ of the profile for equations of the form (4.1). It is an open problem to characterize those nonlinearities for which there is convergence as $t \rightarrow \infty$ of the asymptotic profile towards a fixed state.

\section{An alternative approach to intermediate asymptotics for general nonlinearities}

In this section, we propose an alternative procedure in order to detect the typical asymptotic state for a general nonlinear diffusion equation as fixed points of some renormalized flow map. As we shall see, the present approach will narrow the class of admissible nonlinearities. However, it has the advantage of providing a convergence result of the renormalized solutions towards a stationary profile. Let us consider again the nonlinear diffusion equation

$$
\frac{\partial v}{\partial t}=\Delta f(v)
$$

where $v=v(x, t), x \in \mathbb{R}^{N}, t \geq 0$. As before, $f: \mathbb{R}_{+} \rightarrow \mathbb{R}_{+}$is a continuous and 
(a)
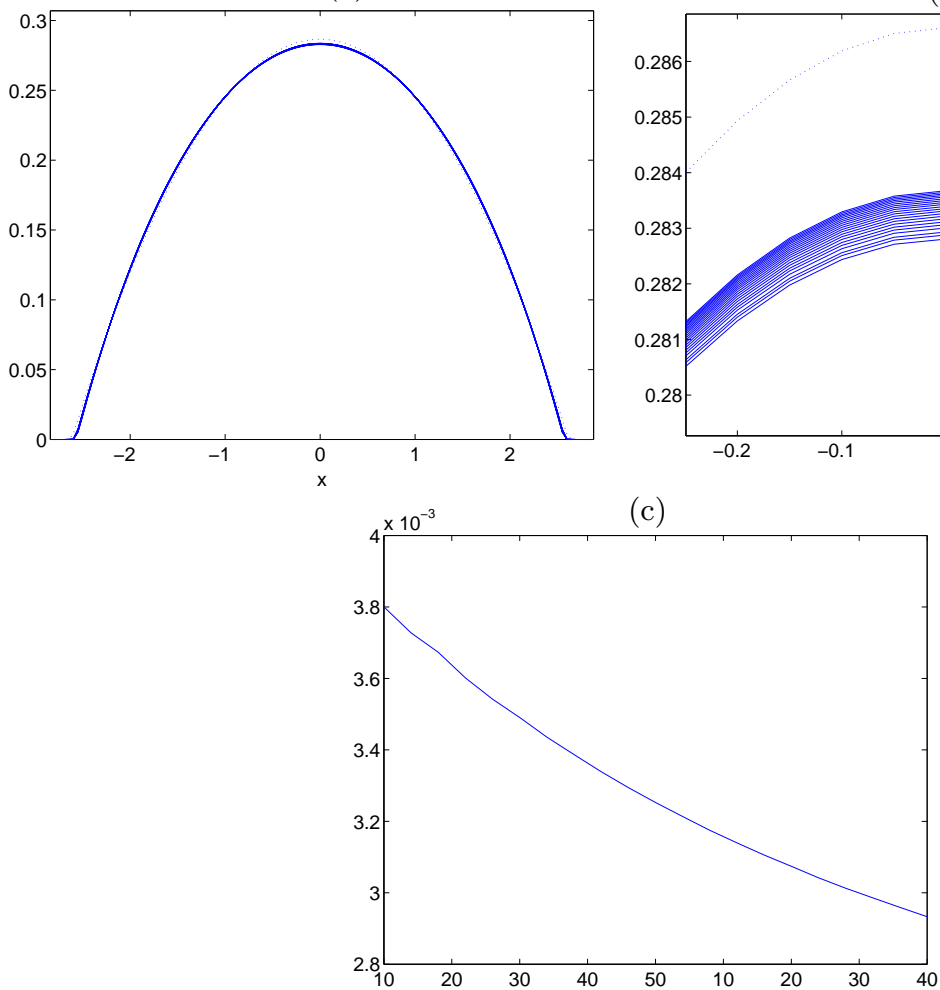

(b)
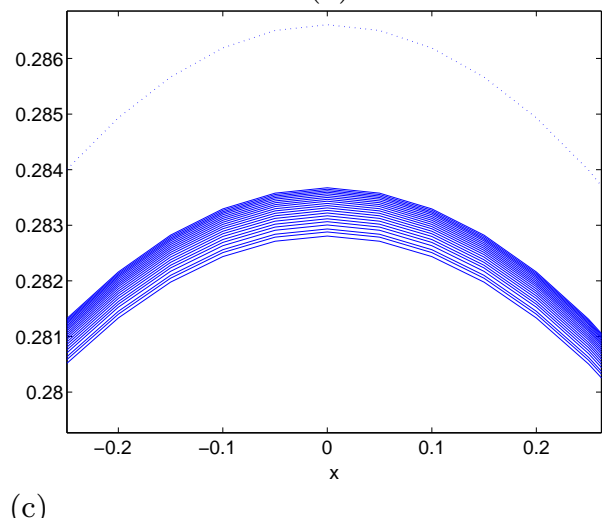

nd $x=0,(c)\left|v_{\infty}(x=0, t)-B(x=0)\right|$.

strictly increasing function. We consider only positive solutions. We denote again the temperature of a solution $v$ at time $t$ by

$$
\theta(v)(t)=\int_{\mathbb{R}^{N}} \frac{|x|^{2}}{2} v(x, t) d x .
$$

The idea of the present approach is to rescale the solution in a similar fashion as for the porous medium case. Of course in general the equation does not enjoy a similarity structure, therefore the choice of the new variables is not trivial. Hereafter we shall require $f$ to satisfy assumptions (NL1)-(NL3)-(FT) defined in the previous section. Moreover, for some positive constant $C$ we require

(NL4) $f^{\prime}(v) \leq C v^{m-1} \quad$ as $0<v \ll 1$,

where $m$ is the same exponent as in assumption (NL2). Under the above hypothesis, we prove that the temperature of the solution $v$ grows like a certain power as $t \rightarrow \infty$.

Lemma 5.1. Let $f: \mathbb{R}_{+} \rightarrow \mathbb{R}_{+}$satisfy (NL1)-(NL4)-(FT). Let $v$ be the solution to (5.1) with initial datum $v_{I} \in L_{+}^{1}\left(\mathbb{R}^{N}\right)$ with unit mass and finite temperature $\theta_{0}$. Then, there exist two positive constants $A_{1}$ and $A_{2}$ such that the temperature $\theta(v)(t)$ of the solution $v(t)$ satisfies

$$
A_{1} t^{\frac{2}{\lambda}} \leq \theta(v)(t) \leq \theta_{0}+A_{2} t^{\frac{2}{\lambda}}
$$


(a)

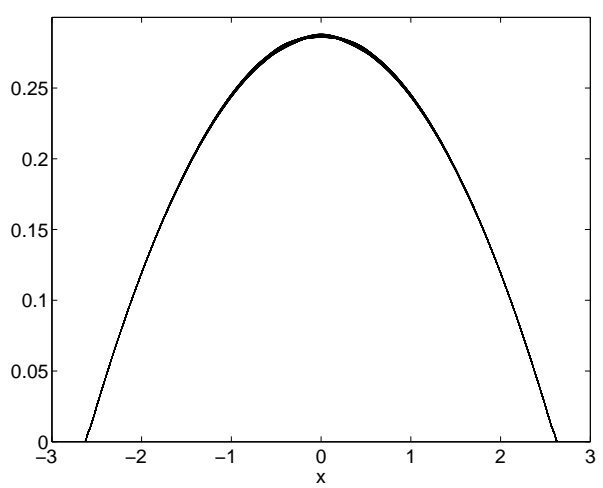

(b)

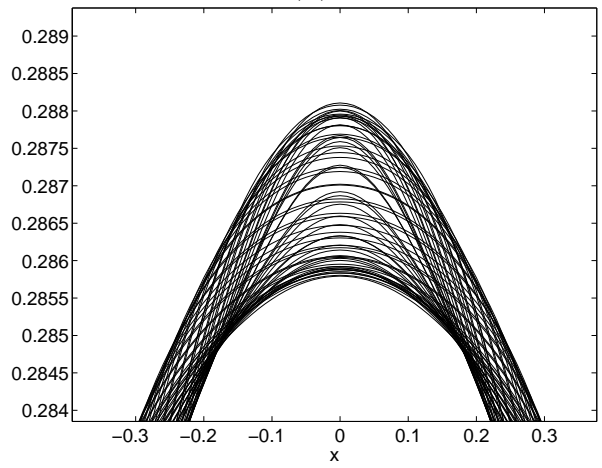

(c)

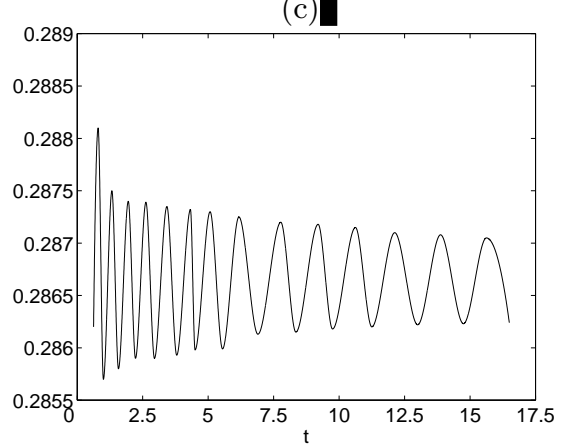

FIG. 4.4. (a) Asymptotic profile of (4.1) where $f$ is defined by (4.6), (b) Zoom of subplot (a) around $x=0,(c) w_{\infty}(x=0, t)$.

for all $t$ larger than a fixed $t_{0}$, where $\lambda=N(m-1)+2$ as usual.

Proof. The first inequality in (5.2) comes from Lemma 4.1, in which (NL2) is used. To prove the second inequality, we use integration by parts to obtain

$$
\begin{aligned}
\frac{d}{d t} \theta(v)(t) & =\int_{\mathbb{R}^{N}} \frac{|x|^{2}}{2} \Delta f(v) d x=-\int_{\mathbb{R}^{N}} x \cdot \nabla f(v) d x=N \int_{\mathbb{R}^{N}} f(v) d x \\
& =N \int_{\mathbb{R}^{N}} \frac{f(v)}{v} v d x \leq N\left\|\frac{f(v)}{v}\right\|_{L^{\infty}} \\
& \leq N \sup _{v \in 0, M t^{-\frac{N}{N(m-1)+2}}}\left|f^{\prime}(v)\right| \leq C(N) t^{-\frac{N(m-1)}{N(m-1)+2}}
\end{aligned}
$$

where we have used (NL4) and the $L^{1}-L^{\infty}$ smoothing effect (see [38]). After integration with respect to the time (5.2) follows.

Let us fix a positive $\theta_{0}$. Let us consider the space of probability measures

$$
\mathcal{M}^{\theta_{0}}=\left\{\mu \in \mathcal{P}_{2}\left(\mathbb{R}^{N}\right), \frac{1}{2} \int|x|^{2} d \mu \leq \theta_{0}\right\},
$$


endowed with the 2 -Wasserstein distance. We define a family of maps $\left\{R_{\alpha}\right\}_{\alpha>\alpha_{0}}$ on the subspace of probability densities of $\mathcal{M}^{\theta_{0}}$, with $\alpha_{0}$ to be chosen later on. For $v_{I} \in L_{1} \cap \mathcal{M}^{\theta_{0}}$ we then set

$$
R_{\alpha} v_{I}(x)=\alpha^{N} v\left(\alpha x, \alpha^{\lambda}\right)
$$

where $v(x, t)$ is the solution to (5.1) with initial datum $v_{I}$. In the following two lemmas we show that $R_{\alpha}$ is a contraction on the metric space $L_{1} \cap \mathcal{M}^{\theta_{0}}$ for all $\alpha>2$.

Lemma 5.2. Let $\theta_{0}>2 A_{2}$ be fixed, where $A_{2}$ is the constant in Lemma 5.1. Then, for any $\alpha>2, R_{\alpha} v_{I} \in L_{1} \cap \mathcal{M}^{\theta_{0}}$.

Proof. It is clear that the scaling (5.3) is mass preserving. We now estimate the temperature of $R_{\alpha} v_{I}$ by means of the estimate (5.2) in Lemma 5.1. We have

$$
\begin{aligned}
\int R_{\alpha} v_{I}(x)|x|^{2} d x=\int \alpha^{N} v\left(\alpha x, \alpha^{\lambda}\right)|x|^{2} d x & =\alpha^{-2} \int v\left(y, \alpha^{\lambda}\right)|y|^{2} d y \\
& \leq \alpha^{-2}\left[\theta_{0}+A_{2} \alpha^{\frac{2 \lambda}{\lambda}}\right]=\theta_{0} \alpha^{-2}+A_{2} .
\end{aligned}
$$

Hence, by choosing $\theta_{0}>2 A_{2}$ and $\alpha>2$ (we recall that the constant $A_{2}$ depends only on the dimension and on the function $f$ ), the proof is complete.

Lemma 5.3. The map $R_{\alpha}: \mathcal{M}^{\theta_{0}} \rightarrow \mathcal{M}^{\theta_{0}}$ is a contraction for all $\alpha>2$.

Proof. Let $v_{I, 1}, v_{I, 2} \in L^{1} \cap \mathcal{M}^{\theta_{0}}$, and let $v_{1}$ and $v_{2}$ be the solutions to (5.1) with initial data $v_{I, 1}$ and $v_{I, 2}$ respectively. We recall the following scaling property of the 2-Wasserstein distance $W_{2}$. Let $\rho_{1}, \rho_{2}$ be two probability densities on $\mathbb{R}^{N}$, and let $\rho_{i}^{\mu}(x)=\mu^{N} \rho_{i}(\mu x), i=1,2, \mu>0$. It holds

$$
W_{2}\left(\rho_{1}^{\mu}, \rho_{2}^{\mu}\right)=\mu^{-1} W_{2}\left(\rho_{1}, \rho_{2}\right) .
$$

The proof of (5.4) is straightforward (see [39, Proposition 7.16]). Using (5.4) we get

$$
W_{2}\left(R_{\alpha} v_{I, 1}, R_{\alpha} v_{I, 2}\right)=\alpha^{-1} W_{2}\left(v_{1}\left(\cdot, \alpha^{\lambda}\right), v_{2}\left(\cdot, \alpha^{\lambda}\right)\right) .
$$

Finally, we use the non-expansive contraction property of the semigroup $v(t)$ with respect to the $p$-Wasserstein distances, which is a consequence of assumption (NL3) (see $[1,29,17])$ to recover

$$
W_{2}\left(R_{\alpha} v_{I, 1}, R_{\alpha} v_{I, 2}\right) \leq \alpha^{-1} W_{2}\left(v_{I, 1}, v_{I, 2}\right),
$$

and the proof is complete.

We now extend the map $R_{\alpha}, \alpha>2$, to the whole space of probability measures $\mathcal{M}^{\theta_{0}}\left(\theta_{0}>2 A_{2}\right)$ by density and uniform continuity with same technique as in [11], so that each map $R_{\alpha}$ is a contraction on a complete metric space. Therefore, we conclude the existence of a family of fixed points $v_{\alpha, \theta_{0}}^{\infty}$ (depending on the parameter $\alpha$ of the map $R_{\alpha}$ and on $\mathcal{M}^{\theta_{0}}$ ).

Finally, we prove that the two parameters family $\left\{v_{\alpha, \theta_{0}}^{\infty}\right\}$ is actually a one parameter family, because we can drop out the dependence on the temperature $\theta_{0}$. To see this, we first observe that each map $R_{\alpha}$ defined on a space $\mathcal{M}^{\theta_{0}}$ depends a priori on $\theta_{0}$ too. However, it easily seen that the action of $R_{\alpha}$ on a function $v_{I}$ does not 
depend on the upper bound $\theta_{0}$ on the temperature of $v_{I}$. Hence, by uniqueness in the Banach fixed point theorem we have, for $\theta_{0} \neq \theta_{1}$,

$$
v_{\alpha, \theta_{0}}^{\infty}=v_{\alpha, \theta_{1}}^{\infty} .
$$

We have thus proven that there exists a one-parameter family $\left\{v_{\alpha}^{\infty}\right\}_{\alpha>2}$ such that

$$
W_{p}\left(\alpha^{N} v\left(\alpha \cdot, \alpha^{\lambda}\right), v_{\alpha}^{\infty}\right) \longrightarrow 0 \quad \text { as } \alpha \rightarrow \infty,
$$

for all solutions $v(x, t)$ to $(5.1)$.

This alternative approach has also the advantage that we can relate the scaled function $\alpha^{N} v\left(\alpha x, \alpha^{\lambda}\right)$ to the solution of a partial differential equation. More precisely, let $v$ be the solution to (5.1) with initial datum $v_{0}$, we have

$$
\alpha^{N} v\left(\alpha x, \alpha^{\lambda}\right)=R_{\alpha} v_{I}(x)=\mathcal{V}_{\alpha}(x, 1),
$$

where $\mathcal{V}_{\alpha}(x, t)$ solves

$$
\left\{\begin{array}{l}
\frac{\partial \mathcal{V}_{\alpha}}{\partial t}=\alpha^{m N} \Delta f\left(\alpha^{-N} \mathcal{V}_{\alpha}\right) \\
\mathcal{V}(x, 0)=\alpha^{N} v_{I}(\alpha x)
\end{array}\right.
$$

This is easily seen by solving the above problem, by performing the scaling

$$
\alpha^{N} v\left(\alpha x, \alpha^{\lambda} t\right)=\mathcal{V}_{\alpha}(x, t),
$$

and by observing that $v$ solves (5.1). Finally, one immediately realizes that $\mathcal{V}_{\alpha}(x, 1)$ is exactly $R_{\alpha} v_{I}(x)$. In the homogeneous case $f(v)=v^{m}$, the equation in the Cauchy problem (5.6) is again the original equation (5.1) (i.e. the porous medium equation). This is due to the well known invariance property of the porous medium equation under its similarity transformation. In such a case, one can prove that the family of fixed points is independent on the parameter $\alpha$ and it coincides with the rescaled Barenblatt profile which is a stationary solution to a nonlinear Fokker-Planck equation.

As a consequence of what we have proved in this section, for any fixed $\alpha$ we have

$$
v_{\alpha}^{\infty}(x)=\mathcal{V}_{\alpha}(x, 1),
$$

where $\mathcal{V}_{\alpha}$ is the solution to (5.6) with initial datum $\alpha^{N} v_{\alpha}^{\infty}(\alpha x)$. Hence, one can try to investigate the limiting behavior of the solutions to (5.6) as $\alpha \rightarrow \infty$ in order to detect an eventual limit as $\alpha \rightarrow \infty$ for the family of fixed points $v_{\alpha}^{\infty}$. In order to perform this task, we use the following strategy. We first analyze the asymptotic behavior with respect to the parameter $\alpha$ of the rescaled solutions $\mathcal{V}_{\alpha}$ under quite general assumptions on the initial data. In particular, under certain extra assumptions on $f$ we shall be able to detect a unique limit point $\mathcal{V}_{\infty}$ (in some sense to be specified afterwards) for the family $\left\{\mathcal{V}_{\alpha}\right\}_{\alpha}$ by means of standard energy techniques. Then, we apply such convergence result to some suitable choice of initial datum $\overline{v_{I}}$ in order to obtain convergence with respect to the $W_{2}$ distance of $R_{\alpha}\left(\overline{v_{I}}\right)$ as $\alpha \rightarrow+\infty$ towards the unique limit point $\mathcal{V}_{\infty}$. Finally, we obtain convergence in $W_{2}$ of $v_{\alpha}^{\infty}$ to $\mathcal{V}_{\infty}$ by means of (5.5) and by triangulation. We remark that the parameter $\alpha$ appears both in the equation in (5.6) and in the rescaled initial datum $\alpha^{N} v_{I}(\alpha \cdot)$, so that we are dealing both with a problem of continuous dependence on the initial data (with initial data eventually approaching a measure, as we will see later) and with a continuous dependence on the nonlinearity function. 
In order to pursue our goal, let us first identify the limit of the rescaled initial data $\mathcal{V}_{\alpha}(\cdot, 0)=\alpha^{N} v_{I}(\alpha \cdot)$ for some $v_{I} \in L_{+}^{1} \cap L^{\infty}$ as $\alpha \rightarrow \infty$. We make use of the following

Lemma 5.4. $\mathcal{V}_{\alpha}(\cdot, 0)=\alpha^{N} v_{I}(\alpha \cdot) \rightarrow \delta_{0}$ as $\alpha \rightarrow \infty$ in the sense of measures.

Next, let us characterize those nonlinearities for which $\alpha^{N m} f\left(\alpha^{-N} v\right)$ admits a limit as $\alpha \rightarrow+\infty$ for fixed $v>0$. Let us then impose

$$
\alpha^{N m} f\left(\alpha^{-N} v\right) \rightarrow \Phi(v), \quad \text { as } \alpha \rightarrow+\infty .
$$

By a change of variable $\alpha^{-N} v=z$, we have

$$
\lim _{z \rightarrow 0} v^{m} \frac{f(z)}{z^{m}}=\Phi(v)
$$

and therefore $f(z) \sim C z^{m}$ as $z \rightarrow 0$ for some constant $C$. Thus, $\Phi(v)=C v^{m}$. This heuristic limit procedure suggests us that we may hope to get some result when passing to the limit at least in case $f$ behaves like a power in zero. Let us then impose the following assumption:

(NL5) $\lim _{v \rightarrow 0} \frac{f(v)-C v^{m}}{v^{m}}=0, \quad$ for some positive constant $C$.

We now aim to prove some strong compactness for the family of functions $\left\{\mathcal{V}_{\alpha}(\cdot, \cdot)\right\}_{\alpha}$ in order to get the limit as $\alpha \rightarrow+\infty$ in a suitable way. To perform this task we generalize the standard energy method for the porous medium equation (see for instance [36]). We shall prove the following lemma.

LEMMA 5.5. For any $0<t_{1}<t_{2}$ and for any $\alpha>2$, we have

$$
\int_{t_{1}}^{t_{2}} \int_{\mathbb{R}^{d}}\left[\left|\frac{\partial}{\partial t} \alpha^{m N} f\left(\alpha^{-N} \mathcal{V}_{\alpha}(x, t)\right)\right|^{2}+\left|\alpha^{m N} \nabla f\left(\alpha^{-N} \mathcal{V}_{\alpha}(x, t)\right)\right|^{2}\right] d x d t \leq C,
$$

for some constant $C$ depending only on the mass, on the function $f$ and on $t_{1}$.

Proof. In what follows we shall assume that the solution $\mathcal{V}_{\alpha}$ to $(5.6)$ is smooth enough to perform the computations below. The assertion above for a general weak solution can be obtained by standard approximation. We first observe that the family of solutions $\mathcal{V}_{\alpha}$ enjoys a bound in $L^{\infty}\left(\left[t_{0},+\infty\right] \times \mathbb{R}^{N}\right)$ which is uniform with respect to $\alpha$. This comes from the usual $L^{1}-L^{\infty}$ regularizing property for nonlinear diffusion, which is a consequence of assumption (NL2) above. Such bound eventually holds in $L^{\infty}\left([0,+\infty] \times \mathbb{R}^{N}\right)$ if the initial data are bounded. Let us multiply the equation in (5.6) by $\alpha^{-N} f\left(\alpha^{-N} \mathcal{V}_{\alpha}\right)$ and integrate over $\mathbb{R}^{N} \times\left[t_{1}, t_{2}\right]$. Integration by parts yields

$$
\alpha^{(m-1) N} \int_{t_{1}}^{t_{2}} \int_{\mathbb{R}^{N}}\left|\nabla f\left(\alpha^{-N} \mathcal{V}_{\alpha}(x, t)\right)\right|^{2} d x d t \leq \int F\left(\alpha^{-N} \mathcal{V}_{\alpha}\left(x, t_{1}\right)\right) d x
$$

where $F(v)=\int_{0}^{v} f(\zeta) d \zeta$. Then, since $f(v) \leq C v^{m}$ as $v \in[0, M]$, we have $F(v) \leq$ $C(m) v^{m+1}$ on the same interval. Thus, due to uniform bound in $L^{\infty}$ for the family $\mathcal{V}_{\alpha}$, we deduce

$$
\alpha^{2 m N} \int_{t_{1}}^{t_{2}} \int_{\mathbb{R}^{N}}\left|\nabla f\left(\alpha^{m N} \mathcal{V}_{\alpha}(x, t)\right)\right|^{2} d x d t \leq C
$$


for some $C$ independent on $\alpha$ and depending on $t_{1}$ and on the initial mass. In order to estimate the time derivative term in (5.7) we compute

$$
\begin{aligned}
& \frac{1}{2} \frac{d}{d t} \int_{\mathbb{R}^{N}}\left|\nabla \alpha^{m N} f\left(\alpha^{-N} \mathcal{V}_{\alpha}\right)\right|^{2}=\alpha^{2 m N} \int_{\mathbb{R}^{N}} \nabla f\left(\alpha^{-N} \mathcal{V}_{\alpha}\right) \cdot \nabla \partial_{t} f\left(\alpha^{-N} \mathcal{V}_{\alpha}\right) d x \\
= & -\alpha^{2 m N} \int_{\mathbb{R}^{N}} \Delta f\left(\alpha^{-N} \mathcal{V}_{\alpha}\right) \partial_{t} f\left(\alpha^{-N} \mathcal{V}_{\alpha}\right) d x=-\alpha^{N(m-1)} \int_{\mathbb{R}^{N}}\left|\partial_{t} \mathcal{V}_{\alpha}\right|^{2} f^{\prime}\left(\alpha^{-N} \mathcal{V}_{\alpha}\right) d x
\end{aligned}
$$

Let us define $G(v)=\int_{0}^{v} f^{\prime}(\zeta)^{1 / 2} d \zeta$. We have, then

$$
\frac{1}{2} \frac{d}{d t} \int_{\mathbb{R}^{N}}\left|\nabla \alpha^{N m} f\left(\alpha^{-N} \mathcal{V}_{\alpha}\right)\right|^{2}=-\int_{\mathbb{R}^{N}}\left|\frac{\partial}{\partial t}\left(\alpha^{N(m+1) / 2} G\left(\alpha^{-N} \mathcal{V}_{\alpha}\right)\right)\right|^{2} d x
$$

Therefore, integration with respect to $t$ over $\left(t_{1} / 2, t_{2}\right)$ yields

$$
\begin{aligned}
\int_{t_{1} / 2}^{t_{2}} \int_{\mathbb{R}^{N}}\left(t-t_{1} / 2\right)\left|\frac{\partial}{\partial t}\left(\alpha^{N(m+1) / 2} G\left(\alpha^{-N} \mathcal{V}_{\alpha}\right)\right)\right|^{2} d x \\
=-\frac{1}{2} \int_{t_{1} / 2}^{t_{2}}\left(t-t_{1} / 2\right) \frac{d}{d t}\left[\int_{\mathbb{R}^{N}}\left|\alpha^{m N} \nabla f\left(\alpha^{-N} \mathcal{V}_{\alpha}(x, t)\right)\right|^{2} d x\right] d t \\
=-\frac{1}{2}\left(t_{2}-\frac{t_{1}}{2}\right) \int_{\mathbb{R}^{N}}\left|\alpha^{m N} \nabla f\left(\alpha^{-N} \mathcal{V}_{\alpha}\left(x, t_{2}\right)\right)\right|^{2} d x \\
+\frac{1}{2} \int_{t_{1} / 2}^{t_{2}} \int_{\mathbb{R}^{N}}\left|\alpha^{N m} \nabla f\left(\alpha^{-N} \mathcal{V}_{\alpha}\right)\right|^{2} d x d t
\end{aligned}
$$

Thanks to (5.8), we can find a constant $C$ depending on $t_{1}$ such that

$$
\int_{t_{1}}^{t_{2}} \int_{\mathbb{R}^{N}}\left|\frac{\partial}{\partial t}\left(\alpha^{N(m+1) / 2} G\left(\alpha^{-N} \mathcal{V}_{\alpha}\right)\right)\right|^{2} d x \leq C
$$

Moreover, assumption (NL4) and the uniform bound in $L^{\infty}$ of $\mathcal{V}_{\alpha}$ imply

$$
\begin{aligned}
\left|\partial_{t} \alpha^{N(m+1) / 2} G\left(\alpha^{-N} \mathcal{V}_{\alpha}\right)\right|^{2} & =\left|\alpha^{\frac{N(m-1)}{2}} G^{\prime}\left(\alpha^{-N} \mathcal{V}_{\alpha}\right) \partial_{t} \mathcal{V}_{\alpha}\right|^{2} \\
& =\left[\alpha^{\frac{N(1-m)}{2}} \frac{G^{\prime}\left(\alpha^{-N} \mathcal{V}_{\alpha}\right)}{f^{\prime}\left(\alpha^{-N} \mathcal{V}_{\alpha}\right)}\right]^{2}\left|\partial_{t}\left(\alpha^{N m} f\left(\alpha^{-N} \mathcal{V}_{\alpha}\right)\right)\right|^{2} \\
& =\alpha^{N(1-m)} f^{\prime}\left(\alpha^{-N} \mathcal{V}_{\alpha}\right)^{-1}\left|\partial_{t}\left(\alpha^{N m} f\left(\alpha^{-N} \mathcal{V}_{\alpha}\right)\right)\right|^{2} \\
& \geq c_{0}\left|\partial_{t}\left(\alpha^{N m} f\left(\alpha^{-N} \mathcal{V}_{\alpha}\right)\right)\right|^{2}
\end{aligned}
$$

for some constant $c_{0}$ depending on the function $f$ and on the initial mass. Therefore we can recover an $L^{2}$-estimate for the time derivative of $\alpha^{m d} f\left(\alpha^{N} \mathcal{V}_{\alpha}(x, t)\right)$ and the proof is completed.

As a consequence of the previous lemma, by Sobolev embedding, we recover

$$
\alpha^{N m} f\left(\alpha^{-N} \mathcal{V}_{\alpha}(x, t)\right) \subset \subset L_{\text {loc }, x, t}^{2}
$$

Now, we prove that assumption (NL5) implies in particular, for all $M>0$,

$$
\lim _{\alpha \rightarrow+\infty} \sup _{0 \leq v \leq M}\left|\alpha^{N m}\left[f\left(\alpha^{-N} v\right)-C \alpha^{-N m} v^{m}\right]\right| \rightarrow 0, \quad \text { as } \quad \alpha \rightarrow+\infty .
$$


To see this, let us fix a positive $\varepsilon>0$. Then, thanks to (NL5) there exists a $\alpha_{0}$ such that

$$
\left|\alpha^{N m}\left[f\left(\alpha^{-N}\right)-C \alpha^{-N m}\right]\right|<\frac{\varepsilon}{M^{m}},
$$

as $\alpha>\alpha_{0}$. Let us set $\alpha_{1}:=\alpha_{0} M^{1 / N}$. Then, for all $\alpha>\alpha_{1}$ and for all $0 \leq v \leq M$ we have $\alpha v^{-1 / N}>\alpha_{0}$, which implies

$$
\left|\alpha^{N m} v^{-m}\left[f\left(\alpha^{-N} v\right)-C \alpha^{-N m} v^{m}\right]\right|<\frac{\varepsilon}{M^{m}} \leq \frac{\varepsilon}{v^{m}},
$$

for all $0 \leq v \leq M$, and this yields

$$
\sup _{0 \leq v \leq M}\left|\alpha^{N m}\left[f\left(\alpha^{-N} v\right)-C \alpha^{-N m} v^{m}\right]\right|<\varepsilon,
$$

for all $\alpha>\alpha_{1}$, which proves (5.9).

Thanks to (5.9) and thanks to the uniform estimate $\left\|\mathcal{V}_{\alpha}(\cdot, t)\right\|_{L^{\infty}} \leq M, M$ depending only on the initial mass (which is a consequence of the $L^{1}-L^{\infty}$ regularizing property), in view of the compactness of $\alpha^{N m} f\left(\alpha^{-N} \mathcal{V}_{\alpha}(x, t)\right)$, we can extract a sequence $\alpha_{n} \rightarrow+\infty$ such that

$$
\left\{\begin{array}{l}
\mathcal{V}_{\alpha_{n}}(\cdot, \cdot) \rightarrow \mathcal{V}_{\infty}, \\
\alpha_{n}^{m d} f\left(\alpha^{-N} \mathcal{V}_{\alpha_{n}}\right) \rightarrow C \mathcal{V}_{\infty}^{m},
\end{array}\right.
$$

almost everywhere in $(x, t)$, for some $\mathcal{V}_{\infty} \in L^{2 m}$, as $n \rightarrow+\infty$. We observe that, in principle, the sequence $\mathcal{V}_{\alpha_{n}}$ may depend on the time interval $\left[t_{1}, t_{2}\right]$. We can construct the desired sequence by standard diagonal procedure.

Finally, we prove that all the limit points of the family $\left\{\mathcal{V}_{\alpha}(x, t)\right\}_{\alpha>2}$ must coincide. Let $\psi$ be a test function on $\left[t_{1}, t_{2}\right] \times \mathbb{R}^{N}$. By definition of distributional solution of the equation in (5.6) we obtain, for all $\alpha>2$,

$-\int_{0}^{T} \int_{\mathbb{R}^{N}} \alpha^{m N} f\left(\alpha^{-N} \mathcal{V}_{\alpha}\right) \Delta \psi d x d t+\int_{\mathbb{R}^{N}} \mathcal{V}_{\alpha}(x, T) \psi(x, T) d x-\int_{\mathbb{R}^{N}} \mathcal{V}_{\alpha}(x, 0) \psi(x, 0) d x=0$.

By taking the limit as $\alpha \rightarrow+\infty$, by means of the dominated convergence theorem and in view of lemma 5.4 and by the uniform bound in $L^{\infty}$ for $\mathcal{V}_{\alpha}$, we obtain

$$
-\int_{0}^{T} \int_{\mathbb{R}^{N}} C \mathcal{V}_{\infty}^{m} \Delta \psi d x d t+\int_{\mathbb{R}^{N}} \mathcal{V}_{\infty}(x, T) \psi(x, T) d x-\delta_{0}(\psi)=0,
$$

which means that $\mathcal{V}_{\infty}$ is the only measure valued solution to the porous medium equation $v_{t}=C \Delta v^{m}$ with a Dirac mass as initial datum, i.e., the corresponding Barenblatt solution (see [30] for uniqueness of solutions with initial datum a measure). Then, we can state the following assertion

$$
\mathcal{V}_{\alpha}(\cdot, \cdot) \rightarrow \mathcal{V}_{\infty}(\cdot, \cdot) \quad \text { almost everywhere in }(0,+\infty) \times \mathbb{R}^{N} .
$$

Then, there exists a $t_{0}$ such that $0<t_{0}<1$ such that

$$
\mathcal{V}_{\alpha}\left(\cdot, t_{0}\right) \rightarrow \mathcal{V}_{\infty}\left(\cdot, t_{0}\right) \quad \text { almost everywhere in } \mathbb{R}^{N} .
$$


Let us then compute the distance between $\mathcal{V}_{\alpha}\left(\cdot, t_{0}\right)$ and $\mathcal{V}_{\infty}\left(\cdot, t_{0}\right)$ in $L^{1}$. For $R>0$ we have

$$
\begin{aligned}
& \int_{\mathbb{R}^{N}}\left|\mathcal{V}_{\alpha}\left(x, t_{0}\right)-\mathcal{V}_{\infty}\left(x, t_{0}\right)\right| d x \\
= & \int_{|x|>R}\left|\mathcal{V}_{\alpha}\left(x, t_{0}\right)-\mathcal{V}_{\infty}\left(x, t_{0}\right)\right| d x+\int_{|x| \leq R}\left|\mathcal{V}_{\alpha}\left(x, t_{0}\right)-\mathcal{V}_{\infty}\left(x, t_{0}\right)\right| d x \\
\leq & \int_{|x| \leq R}\left|\mathcal{V}_{\alpha}\left(x, t_{0}\right)-\mathcal{V}_{\infty}\left(x, t_{0}\right)\right| d x+\frac{1}{R^{2}} \int_{|x|>R}\left|\mathcal{V}_{\alpha}\left(x, t_{0}\right)-\mathcal{V}_{\infty}\left(x, t_{0}\right)\right||x|^{2} d x
\end{aligned}
$$

The first term in the last line above tends to zero as $\alpha \rightarrow+\infty$ by uniform bound in $L^{\infty}$ for the $\mathcal{V}_{\alpha}$ and because of the dominated convergence theorem. The second term is bounded by $2 \theta_{0} / R^{2}$, and therefore it is arbitrarily small for large $R$. This proves that

$$
\left\|\mathcal{V}_{\alpha}\left(\cdot, t_{0}\right)-\mathcal{V}_{\infty}\left(\cdot, t_{0}\right)\right\|_{L^{1}\left(\mathbb{R}^{N}\right)} \rightarrow 0 \quad \text { as } \alpha \rightarrow+\infty .
$$

So far we did not require any extra assumption on the initial datum $v_{I}$ in (5.6) more than $v_{I} \in \mathcal{M}^{\theta_{0}}$. As announced previously, let us then perform the special choice for the initial datum $\bar{v}_{I} \in L_{+}^{1} \cap C_{b}\left(\mathbb{R}^{N}\right), \bar{v}_{I}$ strictly positive and such that $\int_{\mathbb{R}^{N}} \bar{v}_{I}(x)|x|^{4} d x<\infty$. Then, the corresponding rescaled solution $\mathcal{V}_{\alpha}\left(\bar{v}_{I}\right)$ will be continuous with respect to $t$. Moreover, in a similar fashion as in (5.10), we can easily prove that $\mathcal{V}_{\alpha}\left(\bar{v}_{I}\right)$ belongs in $C\left(\left[t_{0},+\infty\right) ; L_{1}\left(\mathbb{R}^{N}\right)\right)$ for any $\alpha>2$, so that we can apply a continuous dependence result stated in [7], by taking $t_{0}$ as initial time. Such results imply that, $\mathcal{V}_{\alpha}$ converges to $\mathcal{V}_{\infty}$ as $\alpha \rightarrow+\infty$ in $C\left(\left[t_{0},+\infty\right) ; L^{1}\left(\mathbb{R}^{N}\right)\right)$. In particular, such convergence holds pointwise at $t=1$, that is

$$
R_{\alpha}\left(\bar{v}_{I}\right) \rightarrow \bar{v}^{\infty}:=\mathcal{V}_{\infty}(\cdot, 1) \quad \text { almost everywhere in } \mathbb{R}^{N} .
$$

Thanks to our choice of $\bar{v}_{I}$, we can perform the following estimate for the fourth moment of $\mathcal{V}_{\alpha}$. Integration by parts and assumption (NL4) yields

$$
\frac{d}{d t} \int_{\mathbb{R}^{N}} \mathcal{V}_{\alpha}(x, t)|x|^{4} d x=C(N) \alpha^{N m} \int_{\mathbb{R}^{N}} f\left(\alpha^{-N} \mathcal{V}_{\alpha}(x, t)\right)|x|^{2} d x \leq C(N) \int_{\mathbb{R}^{N}} \mathcal{V}_{\alpha}^{m}(x, t)|x|^{2} d x .
$$

Then, after a change of variable we have

$$
\int_{\mathbb{R}^{N}} \mathcal{V}_{\alpha}(x, 1)|x|^{4} d x \leq \frac{1}{\alpha^{4}} \int \overline{v_{I}}(x)|x|^{4} d x+C(N) \theta_{0},
$$

which implies a uniform bound of the fourth moment of $R_{\alpha}\left(\overline{v_{I}}\right)$ with respect to $\alpha$. Thanks to this, we can recover a tightness property of the family $R_{\alpha}\left(\overline{v_{I}}\right)$. Namely, for $R>0$ we have

$$
\int_{|x|>R} R_{\alpha}\left(\overline{v_{I}}\right)(x)|x|^{2} d x \leq \frac{1}{R^{2}} \int_{|x|>R} R_{\alpha}\left(\overline{v_{I}}\right)(x)|x|^{4} d x,
$$

and the left-hand side converges to zero uniformly with respect to $\alpha>2$ as $R \rightarrow$ $+\infty$. We recall that such tightness property plus the weak convergence in the sense of measures of the family $R_{\alpha}\left(\overline{v_{I}}\right)$ to $\mathcal{V}_{\infty}(\cdot, 1)$ (which is a trivial consequence of the convergence almost everywhere and of the uniform bound in $L^{\infty}$ of $\mathcal{V}_{\lambda}$ ) are equivalent 
to the convergence of $R_{\alpha}\left(\overline{v_{I}}\right)$ to $\mathcal{V}_{\infty}(\cdot, 1)$ in the $W_{2}$-topology (see [39, Theorem 7.12]). We have thus proven that

$$
W_{2}\left(R_{\alpha}\left(\overline{v_{I}}\right), \bar{v}^{\infty}\right) \rightarrow 0 \quad \text { as } \quad \alpha \rightarrow+\infty .
$$

Finally, (5.5) with $\overline{v_{I}}$ as initial datum together with (5.11) imply $W_{2}\left(v_{\alpha}^{\infty}, \bar{v}^{\infty}\right) \rightarrow 0$ as $\alpha \rightarrow+\infty$, and therefore, by triangulation

$$
\lim _{\alpha \rightarrow+\infty} W_{2}\left(\alpha^{N} v\left(\alpha \cdot \alpha^{\lambda}\right), \bar{v}^{\infty}\right)=0 .
$$

Therefore, we have proven the following

THEOREM 5.6. Given $f$ satisfying assumptions (NL1)-(NL5), for any solution $v(x, t)$ to the Cauchy problem

$$
\left\{\begin{array}{l}
v_{t}=\Delta f(v) \\
v(x, 0)=v_{I}(x)
\end{array}\right.
$$

with $v_{I} \in L_{+}^{1}\left(\mathbb{R}^{N}\right)$ having finite second moment, the following limiting relation holds

$$
W_{2}\left(\alpha^{N} v\left(\alpha \cdot \alpha^{\lambda}\right), \bar{v}^{\infty}\right) \rightarrow 0,
$$

as $\alpha \rightarrow+\infty$, where $\bar{v}^{\infty}$ is the Barenblatt solution to the porous medium equation $v_{t}=C \Delta v^{m}$, with $C$ and $m$ as in assumption (NL5), evaluated at time $t=1$. Moreover $\bar{v}^{\infty}$ is characterized as the unique limit with respect to the $W_{2}$-distance as $\alpha \rightarrow+\infty$ of the family $v_{\alpha}^{\infty}$ of fixed points of the renormalized flow map

$$
v_{I} \mapsto R_{\alpha} v_{I}(x)=\alpha^{N} v\left(\alpha x, \alpha^{\lambda}\right),
$$

with $v(x, t)$ solution to (5.13) with initial datum $v_{I}$.

REMARK 5.7. We remark that, even though the above theorem holds only in case $f$ behaves like a power near zero, nevertheless we do not need to assume any extra hypothesis on the perturbation term $f(v)-C v^{m}$ more than being faster than $v^{m}$ as $v$ tends to 0 . In the existing literature about this topic, as well as in our Theorem 4.3 in the previous section, the perturbation term need to behave like a power $v^{n}$ with $n>m$ (see for instance [8]).

Acknowledgments. The authors acknowledge support from the European IHP network HYKE "Hyperbolic and Kinetic Equations: Asymptotics, Numerics, Applications" HPRN-CT-2002-00282. JAC acknowledges partial support from DGIMEC (Spain) project MTM2005-08024. MPG has been supported by the Deutsche Forschungsgemeinschaft, grant JU 359/5 (Priority Program "Multi-scale Problems") and by the DFG-Project JU 359/3 (Gerhard Hess Award). JAC and MPG have been supported by the DAAD-Acciones Integradas Project. We thank A. Arnold, A. Jüngel and G. Toscani for fruitful discussions.

\section{REFERENCES}

[1] M. Agueh, Existence of solutions to degenerate parabolic equations via the Monge-Kantorovich theory, C. R. Acad. Sci. Paris Sér. A-B, 2002.

[2] L. Ambrosio, N. Gigli and G. Savaré, Gradient flows in Metric Spaces and in the Space of Probability Measures, Lectures in Mathematics ETH Zurich, Birkhäuser Verlag, Basel, 2005. 
[3] A. Arnold and A. Unterreiter, Entropy of discretized Fokker-Planck equations I - temporal semidiscretization, Comp. Math. Appl., 36, 1683-1690, 2003.

[4] D. G. Aronson and P. Bénilan, Régularité des solutions de l'équation des milieux poreux dans $\mathbf{R}^{N}$, C. R. Acad. Sci. Paris Sér. A 288, 103-105, 1979.

[5] D. Benedetto, E. Caglioti and M. Pulvirenti, A kinetic equation for granular media, RAIRO Modél. Math. Anal. Numér., 31, 615-641, 1997.

[6] P. Bénilan, Opérateurs accrétifs et semi-groupes dans les espaces $L^{p},(1 \leq p \leq \infty)$, FranceJapan Seminar, Tokyo, 1976.

[7] P. Bénilan and M. G. Crandall, The continuous dependence on $\varphi$ of solutions of $u_{t}-\Delta \varphi(u)=0$, Indiana Univ. Math. J., 30, 161-177, 1981.

[8] P. Biler, J. Dolbeault and M. J. Esteban, Intermediate asymptotics in $L^{1}$ for general nonlinear diffusion equations, Appl. Math. Lett., 15, 101-107, 2002.

[9] H. Brézis and M. G. Crandall, Uniqueness of solutions of the initial-value problem for $u_{t}-$ $\Delta \varphi(u)=0$, J. Math. Pures et Appl., 58, 153-163, 1979.

[10] E. Carlen and W. Gangbo, Constrained steepest descent in the 2-Wasserstein metric, Annals Math., 157, 807-846, 2003.

[11] J. A. Carrillo, M. Di Francesco and G. Toscani, Intermediate asymptotics beyond homogeneity and self-similarity: long time behavior for $u_{t}=\Delta \phi(u)$, Arch. Ration. Mech. Anal., 180, 127-149, 2006.

[12] J. A. Carrillo, M. Di Francesco and G. Toscani, Strict contractivity of the 2-Wasserstein distance for the porous medium equation by mass-centering, Proceedings of the AMS, to appear, 2006.

[13] J. A. Carrillo, J. Dolbeault, I. Gentil and A. Jüngel, Entropy-Energy inequalities and improved convergence rates for nonlinear parabolic equations, Discrete and Continuous Dynamical Systems-Series B, 6 (5), 1027-1050, 2006.

[14] J. A. Carrillo, M. P. Gualdani and G. Toscani, Finite speed of propagation in porous media by mass transportation methods, C. R. Acad. Sci. Paris, Ser. I, 338, 815-818, 2004.

[15] J. A. Carrillo, M. Gualdani and A. Jüngel, Convergence of Entropy Decay Schemes for nonlinear Fokker-Planck equations, Preprint 2007.

[16] J. A. Carrillo, A. Jüngel, P. Markowich, G. Toscani and A. Unterreiter, Entropy dissipation methods for degenerate parabolic problems and generalized Sobolev inequalities, Monatsh. Math., 133, 1-82, 2001.

[17] J. A. Carrillo, R. J. McCann and C. Villani, Contractions in the 2-Wasserstein length space and thermalization of granular media, Arch. Rational Mech. Anal., 179, 217-263, 2006.

[18] J. A. Carrillo and G. Toscani, Asymptotic $L^{1}$-decay of solutions of the porous medium equation to self-similarity, Indiana Univ. Math. J., 49, 113-142, 2000.

[19] J. A. Carrillo and G. Toscani, Wasserstein metric and large-time asymptotics of nonlinear diffusion equation, New Trends in Mathematical Physics (In Honour of the Salvatore Rionero 70th Birthday), 240-254, 2005.

[20] J. A. Carrillo and J. L. Vázquez, Asymptotic Complexity in Filtration Equations, Journal of Evolution Equations, preprint, 2007.

[21] J. Dolbeault and M. del Pino, Best constants for Gagliardo-Nirenberg inequalities and applications to nonlinear diffusions, J. Math. Pures Appl., 81, 847-875, 2002.

[22] L. Gosse and G. Toscani, Identification of asymptotic decay to self-similarity for onedimensional filtration equations, SIAM J. Numer. Anal., 43, 2590-2606, 2006.

[23] R. Jordan, D. Kinderlehrer and F. Otto, The variational formulation of the Fokker-Planck equation, SIAM J. Math. Anal., 29, 1-17, 1998.

[24] A. Jüngel, Numerical approximation of a drift-diffusion model for semiconductors with nonlinear diffusion, ZAMM, 75, 783-799, 1995.

[25] A. S. Kalashnikov, Some problems of the qualitative theory of second-order nonlinear degenerate parabolic equations, Russian Math. Surveys, 42, 169-222, 1987.

[26] B. F. Knerr, The porous medium equation in one space dimension, Trans. Amer. Math. Soc., 234, 171-183, 1987.

[27] P. Markowich and C. Villani, On the trend to equilibrium for the Fokker-Planck equation: an interplay between physics and functional analysis, Proceedings, VI Workshop on Partial Differential Equations, Part II (Rio de Janeiro, 1999). Mat. Contemp., 19, 1-29, 2000.

[28] R. J. McCann, A convexity principle for interacting gases, Adv. Math., 128, 153-179, 1997.

[29] F. Otto, The geometry of dissipative evolution equations: the porous medium equation, Comm. Partial Differential Equations, 26, 101-174, 2001.

[30] M. Pierre, Uniqueness of the solutions of $u_{t}-\Delta \phi(u)=0$ with initial datum a measure, Nonlinear Anal., 6, 175-187, 1982.

[31] G. Toscani, Kinetic and hydrodynamical models of nearly elastic granular flows, Monatshefte 
für Mathematik, 142, 179-192, 2004.

[32] G. Toscani, A central limit theorem for solutions of the porous medium equation, J. Evol. Equ., 5, 185-203, 2005.

[33] A. Unterreiter, A. Arnold, P. M. Markowich and G. Toscani, On generalized Csiszár-Kullback inequalities, Monatsh. Math., 131, 235-253, 2000

[34] J. L. Vázquez, Asymptotic behavior and propagation properties of the one-dimensional flow of gas in a porous medium, Trans. Amer. Math. Soc., 277, 507-527, 1983.

[35] J. L. Vázquez, An introduction to the mathematical theory of the porous medium equation, Shape Optimization and Free Boundaries (Montreal, PQ, 1990), NATO Adv. Sci. Inst. Ser. C Math. Phys. Sci., Kluwer Acad. Publ., Dordrecht, 380, 347-389, 1992.

[36] J. L. Vázquez, Asymptotic behavior for the porous medium equation posed in the whole space, J. Evol. Equ., 3, 67-118, 2003

[37] J. L. Vázquez, The porous medium equation: new contractivity results, in Elliptic and parabolic problems, Progr. Nonlinear Dierential Equations Appl., Birkhäuser, Basel, 63, 433-451, 2005.

[38] L. Véron, Effets régularisants de semi-groupes non linéaires dans des espaces de Banach, Ann. Fac. Sci. Toulouse Math., 1, 171-200, 1979.

[39] C. Villani, Topics in optimal mass transportation, Graduate Studies in Mathematics, Amer. Math. Soc., Providence, RI, 58, 2003. 